\title{
Nonlinear chiral transport from holography
}

\author{
Yanyan Bu, ${ }^{a}$ Tuna Demircik ${ }^{b}$ and Michael Lublinsky ${ }^{b}$ \\ ${ }^{a}$ Department of Physics, Harbin Institute of Technology, \\ Harbin 150001, China \\ ${ }^{b}$ Department of Physics, Ben-Gurion University of the Negev, \\ Beer-Sheva 84105, Israel \\ E-mail: yybu@hit.edu.cn, demircik@post.bgu.ac.il, lublinm@bgu.ac.il
}

\begin{abstract}
Nonlinear transport phenomena induced by the chiral anomaly are explored within a $4 \mathrm{D}$ field theory defined holographically as $\mathrm{U}(1)_{V} \times \mathrm{U}(1)_{A}$ Maxwell-Chern-Simons theory in Schwarzschild- $A d S_{5}$. First, in presence of external electromagnetic fields, a general form of vector and axial currents is derived. Then, within the gradient expansion up to third order, we analytically compute all (over 50) transport coefficients. A wealth of higher order (nonlinear) transport phenomena induced by chiral anomaly are found beyond the Chiral Magnetic and Chiral Separation Effects. Some of the higher order terms are relaxation time corrections to the lowest order nonlinear effects. The charge diffusion constant and dispersion relation of the Chiral Magnetic Wave are found to receive anomalyinduced non-linear corrections due to e/m background fields. Furthermore, there emerges a new gapless mode, which we refer to as Chiral Hall Density Wave, propagating along the background Poynting vector.
\end{abstract}

KEYwords: AdS-CFT Correspondence, Gauge-gravity correspondence, Holography and quark-gluon plasmas

ARXIV EPRINT: 1807.08467 


\section{Contents}

1 Introduction 1

2 Summary of the results $\quad 5$

2.1 Generalities 5

2.2 Main results 8

2.2.1 General form of the currents 8

2.2.2 Second order results: comparison with the CKT 9

$\begin{array}{ll}\text { 2.2.3 The third order results and collective excitations } & 12\end{array}$

3 Holographic setup: $\mathrm{U}(1)_{V} \times \mathrm{U}(1)_{A} \quad 14$

4 Nonlinear chiral transport $\quad 17$

$\begin{array}{llr}5 & \text { Conclusion } & 20\end{array}$

$\begin{array}{ll}\text { A Supplement for section } 4 & 21\end{array}$

\section{Introduction}

Hydrodynamics $[1,2]$ is an effective low energy description of many interacting QFTs near thermal equilibrium. Historically, hydrodynamics has been always associated with a long wavelength limit of the underlying microscopic theory, while over the last decade or so there is an increased number of works addressing "hydronization" relaxing the long wavelength approximation. Rather, hydrodynamics is defined as an effective theory of conserved currents, such as stress tensor and/or charge currents, assuming their algebra is closed on a relevant set of near-equilibrium states.

Dynamics of the theory is governed by conservation equations (continuity equations) of the currents. The simplest example is $\partial_{t} \rho=-\vec{\nabla} \cdot \vec{J}$, which is a time evolution equation for the charge density $\rho$ sourced by three-current $\vec{J}$. However, this equation cannot be solved as an initial value problem without additional input, the current $\vec{J}$. In hydrodynamics, $\vec{J}$ has to be expressed in terms of thermodynamical variables, such as $\rho$ itself, temperature, and possibly external fields if present. This is known as constitutive relation. Traditionally, in the long wavelength limit, constitutive relations are presented as a (truncated) gradient expansion. At any given order, this expansion is fixed by thermodynamic considerations and symmetries, up to a finite number of transport coefficients (TCs). The latter should be either computed from underlying microscopic theory or deduced experimentally. Diffusion constant, DC conductivity or shear viscosity are examples of the lowest order TCs.

It is well known, however, that in relativistic theory truncation of the gradient expansion at any fixed order leads to serious conceptual problems such as violation of causality. 
Beyond conceptual issues, causality violation results in numerical instabilities rendering the entire framework unreliable. Causality is restored when all order gradient terms are included, in a way providing a UV completion to the "old" hydrodynamic effective theory. Below we will refer to such case as all order resummed hydrodynamics [3-8]. The first completion of the type was originally proposed by Müller, Israel, and Stewart (MIS) [9-12] who introduced retardation effects in the constitutive relations for the currents. The MIS formulation [9-12] is the most popular scheme employed in practical simulations. Recent ideas on the nature of the hydrodynamic expansion, gradient resummation and attractor behavior, etc. could be found in [4, 13-23].

In this paper we continue exploring hydrodynamic regime of relativistic plasma with chiral asymmetries. We closely follow previous works [24, 25] focusing on massless fermion plasma with two Maxwell gauge fields, $\mathrm{U}(1)_{V} \times \mathrm{U}(1)_{A}$. As a result of chiral anomaly, which appears in relativistic QFTs with massless fermions, global $\mathrm{U}(1)_{A}$ current coupled to external electromagnetic fields is no longer conserved. The continuity equations turn into

$$
\partial_{\mu} J^{\mu}=0, \quad \partial_{\mu} J_{5}^{\mu}=12 \kappa \vec{E} \cdot \vec{B},
$$

where $J^{\mu} / J_{5}^{\mu}$ are vector/axial currents and $\kappa$ is an anomaly coefficient $\left(\kappa=e N_{c} /\left(24 \pi^{2}\right)\right.$ for $\mathrm{SU}\left(N_{c}\right)$ gauge theory with a massless Dirac fermion in fundamental representation and $e$ is electric charge, which will be set to unit from now on). $\vec{E}$ and $\vec{B}$ are vector electromagnetic fields. Non-conservation of the axial current in (1.1) receives extra contribution if external axial electromagnetic fields are turned on. Throughout this work, however, we will not consider external axial fields (they were considered in ref. [24]). Chiral plasma plays a major role in a number of fundamental research areas, historically starting from primordial plasma in the early universe [26-30]. During the last decade, macroscopic effects induced by the chiral anomaly were found to be of relevance in relativistic heavy ion collisions [31-33], and have been searched intensively at LHC [34-38]. Finally, (pseudo-)relativistic systems in condensed matter physics, such as Dirac and Weyl semimetals, display anomaly-induced phenomena, which were recently observed experimentally [39-45] and can be studied via similar theoretical methods [46-49].

A hydrodynamic description of (chiral) plasma amounts to solving a set of coupled equations. As has been mentioned earlier, the continuity equations (1.1) have to be supplemented by constitutive relations describing plasma medium effects. Generically, these are of the type

$$
\vec{J}=\vec{J}\left[\rho, \rho_{5}, T, \vec{E}, \vec{B}\right] ; \quad \vec{J}_{5}=\vec{J}_{5}\left[\rho, \rho_{5}, T, \vec{E}, \vec{B}\right],
$$

where $\rho_{5}$ is the axial charge density and $T$ stands for the temperature. ${ }^{1}$

In a sense, the constitutive relations (1.2) are "off-shell" relations, because they treat the charge density $\rho\left(\rho_{5}\right)$ as independent of $\vec{J}\left(\vec{J}_{5}\right)$. Employing (1.1), the currents (1.2) are put into "on-shell". In (1.2), the fields $\vec{E}, \vec{B}$ are assumed to be external. However, the charges and currents induce $\mathrm{e} / \mathrm{m}$ fields of their own. Thus, the external electromagnetic

\footnotetext{
${ }^{1}$ We prefer to parameterise the currents (1.2) in terms of the charge densities $\rho, \rho_{5}$ because it is more natural and straightforward within the holographic framework. Yet, we could switch to a more traditional representation with the chemical potentials $\mu, \mu_{5}$ as hydrodynamical variables (see section 2.2 for details).
} 
fields $\vec{E}, \vec{B}$ have to be promoted into dynamical ones, satisfying Maxwell equations (in Gaussian units), ${ }^{2}$

$$
\begin{array}{ll}
\vec{\nabla} \cdot \vec{E}=4 \pi \rho^{\mathrm{tot}}, & \vec{\nabla} \times \vec{B}=\frac{1}{c}\left(4 \pi \vec{J}^{\mathrm{tot}}+\partial_{t} \vec{E}\right), \\
\vec{\nabla} \cdot \vec{B}=0, & \vec{\nabla} \times \vec{E}=-\partial_{t} \vec{B},
\end{array}
$$

where $\rho^{\text {tot }}$ and $\vec{J}^{\text {tot }}$ are the total charge density and total current, a sum of external sources $\left(\rho^{\text {ext }}, \vec{J}^{\text {ext }}\right)$ and induced part $(\rho, \vec{J})$, which is the one that enters the constitutive relations (1.2). The external sources could be absent when a fully isolated system is considered. A typical example would be primordial plasma in the early Universe frequently studied using magneto-hydrodynamics (MHD). MHD, along with many other effective theories of the type, also involves neutral flow dynamics. That is, in addition to the charge current sector discussed above, one has to simultaneously consider energy-momentum conservation. Generically, the two dynamical sectors are coupled. However, in the discussion below, we will consider the probe limit, under which one ignores back-reaction of the charge sector on the energy-momentum conservation. This implies $\varepsilon+p \gg \mu \rho+\mu_{5} \rho_{5}$ with $\varepsilon, p$ being the fluid's energy density and pressure.

A self-consistent evolution of the system is determined by solving together (1.1), (1.2), (1.3) given some initial conditions. While the equations (1.1), (1.3) are exact, the constitutive relations (1.2) are the ones where various hydrodynamic approximations are applied. A great deal of modelling normally enters (1.2), such as truncated gradient expansion, weak field approximation, etc. As a result of a full simulation, one sometimes finds instabilities leading to exponential growths of some quantities, such as of dynamical magnetic fields. It thus becomes mandatory to check if the original approximations made for the constitutive relations are consistent with the solutions found. If not, the hydrodynamical model has to be revised.

We just outlined a general setup for a hydrodynamical problem, but it is not our goal here to carry it over for any realistic system. Instead, motivated by the discussion above we would like to focus on the nature of the constitutive relations (1.2), which are well known to receive contributions induced by the chiral anomaly. The most familiar example is the chiral magnetic effect (CME) [50, 51]: a vector current is generated along an external magnetic field when a chiral imbalance between left- and right-handed fermions is present $\left(\vec{J} \sim \rho_{5} \vec{B}\right)$. There is a vast literature on CME, which we cannot review here in full. The chiral magnetic conductivity was computed in perturbative QCD in [52-57]. In [58-74] it was evaluated for the strong coupling regime using AdS/CFT correspondence [75-77]. CME emerged via arguments based on the second law of thermodynamics, that is positivity of entropy production [78, 79], and also within the chiral kinetic theory (CKT) [80-84]. Finally, numerical evidence based on lattice gauge theory for CME can be found in [85-90]. We would like to comment by passing that $\mathrm{CME}$ is believed to be a strict non-equilibrium

\footnotetext{
${ }^{2}$ In principle, the axial sources $\left(\rho_{5}, \overrightarrow{J_{5}}\right)$, through another set of chiral anomaly-modified Maxwell's equations, would also generate classical axial $\mathrm{e} / \mathrm{m}$ fields. In their turn, the axial $\mathrm{e} / \mathrm{m}$ fields would enter and modify the constitutive relations (1.2), see e.g. [24].
} 
phenomenon. In other words, different arguments indicate that CME must vanish in equilibrium $[32,46,91,92]^{3}$

Another important transport phenomenon induced by the chiral anomaly is the chiral separation effect (CSE) [93, 94]: left and right charges get separated along applied external magnetic field $\left(\vec{J}_{5} \sim \vec{B}\right)$. Combined, CME and CSE lead to a new gapless excitation called chiral magnetic wave (CMW) [95]. This is a propagating wave along the magnetic field. While signature of CME/CSE has not yet been confirmed in heavy ion collision experiments [34-37], a large negative longitudinal magneto-resistance observed in Dirac/Weyl semimetals can be attributed to CME [43-45].

Just like in refs. [24, 25], our playground will be a holographic model, namely $\mathrm{U}(1)_{V} \times$ $\mathrm{U}(1)_{A}$ Maxwell-Chern-Simons theory in Schwarzschild- $A d S_{5}[59,64]$ to be introduced in detail in section 3. For some sort of universality, we hope to learn from this model about both generic structures of the currents and relative strengths of various effects.

Recently, transport phenomena nonlinear in external fields were realised [102] to be of critical importance in having self-consistent evolution of chiral plasma. Combined with the causality arguments mentioned earlier, the conclusion is that the constitutive relations (1.2) should contain some "nonlinear" transport coefficients so to guarantee their applicability in a broader regime. Particularly, traditional MHD is strongly affected by anomalous transports [97-101], which necessitates a development of a fully self-consistent chiral MHD. This triggered strong interest in nonlinear chiral transport phenomena within CKT [103-106], to which we will compare some of our findings below. Previous works on the subject of nonlinear anomalous transports include [107] based on the entropy current approach and [108] based on the fluid-gravity correspondence.

The main objective of the series of publications $[24,25,96]$ and the present work is to explore the constitutive relations (1.2) under various approximations, primarily zooming on transport phenomena induced by the chiral anomaly. In the present publication, the following new directions are explored. First, we derive general expressions for the vector and axial currents, see (2.11), (2.12), which do not involve any approximations. This clarifies the concept of "non-renormalisation" of CME/CSE [24, 25, 109, 110] when electromagnetic fields can be both strong and inhomogeneous in spacetime. Second,within the holographic model, we complete the calculation of all second order nonlinear transport coefficients and compare with those obtained in CKT [104]. Finally, and this is the main novel part in this publication, all third order transport coefficients are computed analytically, including relaxation time corrections to some second order transport terms (see section 4). This paves a way for the gradient resummation project released in [96]: some of the third order transport coefficients become all order frequency/momentum-dependent functions.

In the next section, we will review our results including connections to the previous works $[24,25]$ and the forthcoming publication [96]. The remaining sections present details of the calculations.

\footnotetext{
${ }^{3}$ We thank Mikhail Zubkov for bringing this issue to our attention. We also thank Dmitri Kharzeev, Shu Lin, Andrey Sadofyev, and Ho-Ung Yee for stimulating discussions about this point.
} 


\section{Summary of the results}

\subsection{Generalities}

This subsection briefly summarises the series of works $[24,25,96,111]$ including the present one, so to help the reader to navigate between various studies and results. We write down the most comprehensive constitutive relation and indicate specific approximations applied in each individual work.

Following $[24,25]$, the charge densities and external fields are split into constant backgrounds and space-time dependent fluctuations

$$
\begin{aligned}
\rho\left(x_{\alpha}\right) & =\bar{\rho}+\epsilon \delta \rho\left(x_{\alpha}\right), & \rho_{5}\left(x_{\alpha}\right) & =\bar{\rho}_{5}+\epsilon \delta \rho_{5}\left(x_{\alpha}\right), \\
\vec{E}\left(x_{\alpha}\right) & =\overrightarrow{\mathbf{E}}+\epsilon \delta \vec{E}\left(x_{\alpha}\right), & \vec{B}\left(x_{\alpha}\right) & =\overrightarrow{\mathbf{B}}+\epsilon \delta \vec{B}\left(x_{\alpha}\right),
\end{aligned}
$$

where $\bar{\rho}, \bar{\rho}_{5}, \overrightarrow{\mathbf{E}}$ and $\overrightarrow{\mathbf{B}}$ are the backgrounds, while $\delta \rho, \delta \rho_{5}, \delta \vec{E}$ and $\delta \vec{B}$ stand for the fluctuations. Here $\epsilon$ is a formal expansion parameter to be used below. Furthermore, being unable to perform calculations for arbitrary background fields for most of the time, we introduce an expansion in the field strengths

$$
\overrightarrow{\mathbf{E}} \rightarrow \alpha \overrightarrow{\mathbf{E}}, \quad \overrightarrow{\mathbf{B}} \rightarrow \alpha \overrightarrow{\mathbf{B}},
$$

where $\alpha$ is a corresponding expansion parameter. Below we will introduce yet another expansion parameter $\lambda$, which will correspond to the hydrodynamical gradient expansion. For the purpose of gradient counting, e/m fields will be frequently considered as $\mathcal{O}\left(\lambda^{1}\right)$.

The constitutive relations (1.2) can be formally Taylor expanded in all its arguments. This includes the gradient $(\lambda), \epsilon$, and $\alpha$ expansions. Parametrically, a generic term entering (1.2) looks like

$$
\bar{\rho}^{k} \bar{\rho}_{5}^{k_{5}} \overrightarrow{\mathbf{E}}^{n_{E}} \overrightarrow{\mathbf{B}}^{n_{B}} \partial_{t}^{m_{t}} \vec{\nabla}^{m_{x}}\left(\delta \rho^{l} \delta \rho_{5}^{l_{5}} \delta \vec{E}^{l_{E}} \delta \vec{B}^{l_{B}}\right)
$$

which is multiplied by a transport coefficient. ${ }^{4} k, k_{5}, n_{E}, n_{B}, m_{t}, m_{x}, l, l_{5}, l_{E}, l_{B}$ are integers. The most general constitutive relations correspond to a sum of all possible terms like $(2.3) .^{5}$

Obviously, we do not intend to consider all possible terms in (2.3). Instead, most of the results obtained in the present and early works [24, 25] can be combined in a compact constitutive relation (focusing on the vector current $\vec{J}$ ),

$$
\begin{aligned}
\vec{J}= & \gamma_{1} \vec{\nabla} \rho+\gamma_{2} \vec{\nabla} \rho_{5}+\gamma_{3} \vec{E}+\gamma_{4}\left(\rho_{5} \vec{B}\right)+\gamma_{5} \vec{\nabla} \times \vec{B}+\gamma_{6}(\vec{E} \times \vec{\nabla} \rho)+\gamma_{7} \vec{B} \times(\rho \vec{\nabla} \rho) \\
& +\gamma_{18} \vec{B} \times\left(\rho_{5} \vec{\nabla} \rho_{5}\right)+\gamma_{8}\left(\vec{E} \times \vec{\nabla} \rho_{5}\right)+\gamma_{9}(\rho \vec{E} \times \vec{B})+\gamma_{10} \vec{\nabla}\left(\vec{B} \cdot \vec{\nabla} \rho_{5}\right)+\gamma_{11} \vec{\nabla}(\vec{B} \cdot \vec{\nabla} \rho) \\
& +\gamma_{12}\left(\rho \vec{\nabla} B^{2}\right)+\gamma_{13}(\rho \vec{B})+\gamma_{14} \vec{\nabla}(\vec{E} \cdot \vec{\nabla} \rho)+\gamma_{15}(\rho \vec{E})+\gamma_{16} \vec{\nabla}\left(\vec{E} \cdot \vec{\nabla} \rho_{5}\right)+\gamma_{17}\left(\rho_{5} \vec{E}\right) .
\end{aligned}
$$

\footnotetext{
${ }^{4}$ In fact, each term in (2.3) corresponds to a large number of terms obtained by different actions of the derivatives and index contractions.

${ }^{5}$ The asymptotic nature of the gradient expansion and problems related to resummation of the series have been a hot topic over the last few years, see recent works $[13,14,112]$. In our approach, however, we never attempt to actually sum the series and thus these discussions are of no relevance to our formalism.
} 
The coefficients $\gamma_{i}$ are most general $O(3)$ scalars which could be constructed from three vectors $\vec{\nabla}, \vec{E}$, and $\vec{B}$. That is, $\gamma_{i}$ are scalar functions of $E^{2}$ and $B^{2}$, and pseudo-scalar functions of $\vec{E} \cdot \vec{B}$. Furthermore, $\gamma_{i}$ are scalar functionals of derivative operators $\partial_{t}, \vec{\nabla}^{2}$, $\vec{E} \cdot \vec{\nabla}$, and pseudo-scalar functionals of $\vec{B} \cdot \vec{\nabla}$,

$$
\gamma_{i}=\gamma_{i}\left(\partial_{t}, \vec{\nabla}^{2}, \vec{E} \cdot \vec{\nabla}, \vec{B} \cdot \vec{\nabla} ; E^{2}, B^{2}, \vec{E} \cdot \vec{B}\right),
$$

which correspond to all order gradient resummation, as mentioned in Introduction. $\gamma_{i}$ themselves are rich in structure and contain information about non-linear corrections in the fields. Taylor expanding $\gamma_{i}$ in all their arguments (all the derivatives are assumed to act on the right of $\gamma_{i}$ ) gives rise to each individual gradient term like (2.3). Admittedly, (2.4) does not contain all the possible terms like in (2.3). Particularly, while the constitutive relation (2.4) does contain some nonlinear in $\rho, \rho_{5}$ terms, it excludes most of the nonlinear terms of the third order, which are collected in (4.6), (4.7). Some of the terms in (2.4) are well recognisable, such as diffusion $\left(\gamma_{1}\right)$, electrical conductivity $\left(\gamma_{3}\right)$, or CME $\left(\gamma_{4}\right)$. Some other terms might be less familiar and we will discuss them below in detail.

As explained in the Introduction, the purpose of $[24,25,96]$ and the present work is to systematically explore (2.3) under different approximations. We first briefly summarise them using both the notations of (2.3) and (2.4), and then deepen our presentation of the current study.

- Ref. [24], study 1. No background fields, $\overrightarrow{\mathbf{E}}=\overrightarrow{\mathbf{B}}=0$; all order gradient terms that are linear in the inhomogeneous fluctuations $\delta \rho, \delta \rho_{5}, \delta \vec{E}, \delta \vec{B}$ are resummed. ${ }^{6}$ This corresponds to calculating currents up to $\mathcal{O}\left(\epsilon^{1} \alpha^{0}\right)$. Using the notations (2.3) and (2.4) this study corresponds to

$$
\begin{array}{ll}
\underline{[24]-1_{a}:}: \quad n_{E}=n_{B}=0, l+l_{5}+l_{E}+l_{B}=1, \forall\left(k, k_{5}\right), m_{t}+m_{x} \leq 3 \Rightarrow \text { (analytic) } \\
\underline{[24]-1_{n}}: \quad \\
n_{E}=n_{B}=0, l+l_{5}+l_{E}+l_{B}=1, \forall\left(k, k_{5}\right), \forall\left(m_{t}, m_{x}\right) \Rightarrow \text { (numeric) } \\
\gamma_{i}=\gamma_{i}\left(\partial_{t}, \vec{\nabla}, 0,0 ; 0,0,0\right), \quad i=1,3,4,5 .
\end{array}
$$

The remaining $\gamma_{i}$ have not been probed in the study. $\gamma_{i}\left(\partial_{t}, \vec{\nabla}\right)$ correspond to the gradient resummation. Thanks to the linearisation, the constitutive relations could be conveniently expressed in Fourier space. Then, the functionals of the derivatives are turned into functions of frequency and space momenta, $\left(\partial_{t}, \vec{\nabla}\right) \rightarrow(-i \omega, i \vec{q})$. We refer to $\gamma_{i}\left(-i \omega, q^{2}\right)$ as transport coefficients functions (TCFs) [6]. TCFs contain information about infinitely many derivatives and associated transport coefficients. In practice, TCFs are not computed as a series resummation of order-by-order hydrodynamic expansion, and are in fact exact to all orders. TCFs go beyond the hydrodynamic low frequency/momentum limit and contain collective effects of non-hydrodynamic modes. Fourier transformed back into real space, TCFs become memory functions. Diffusion and shear viscosity memory functions were previously computed in $[7,111]$.

\footnotetext{
${ }^{6}$ In [24] we also considered transports related to axial external electromagnetic fields.
} 
- Ref. [24], study 2. Nonlinear in $\overrightarrow{\mathbf{E}}$ and $\overrightarrow{\mathbf{B}}$ corrections to the vector/axial currents. The currents are derived up to $\mathcal{O}\left(\epsilon^{0} \alpha^{3}\right)$.

$$
\begin{aligned}
\underline{[24]-2}: \quad n_{E}+n_{B} & \leq 3, l=l_{5}=l_{E}=l_{B}=m_{t}=m_{x}=0, \forall\left(k, k_{5}\right) \Rightarrow \text { (analytic) } \\
\gamma_{i} & =\gamma_{i}\left(0,0,0,0 ; E^{2}, B^{2}, \vec{E} \cdot \vec{B}\right), \quad i=3,4,9 .
\end{aligned}
$$

- Ref. [25], study 1. Nonlinear corrections to vector/axial currents due to static but spatially-inhomogeneous magnetic field.

$$
\begin{aligned}
\underline{[25]-1_{a}:}: & n_{E}=l_{E}=0, \forall\left(l, l_{5}, k, k_{5}\right), n_{B}+m_{t}+m_{x}+l_{B} \leq 2 \Rightarrow \text { (analytic) } \\
\underline{[25]-1_{a}}: \quad & n_{E}=l_{E}=0, \forall\left(l, l_{5}, k, k_{5}\right), n_{B}+m_{t}+m_{x}+l_{B}=3, l+l_{5}+k+k_{5}=1 \\
& \Rightarrow(\text { analytic }) \\
\gamma_{1,4} & =\gamma_{1,4}\left(\partial_{t}, 0,0,0 ; E^{2}, 0,0\right), \quad \gamma_{5}=\gamma_{5}(0,0,0,0 ; 0,0,0), \\
\gamma_{7,18} & =\gamma_{7,18}(0,0,0,0 ; 0,0,0), \quad \gamma_{10,11,12}=\gamma_{10,11,12}(0,0,0,0 ; 0,0,0) .
\end{aligned}
$$

- Ref. [25], study 2. Dependence of longitudinal electric conductivity on arbitrary strong constant magnetic field. A time-varying electric field is assumed to be weak.

$\underline{[25]-2_{a}}: \quad n_{E}=l_{B}=l=k=k_{5}=m_{x}=0, \forall\left(n_{B}, l_{5}, l_{E}\right), m_{t}+n_{B}+l_{E} \leq 3, \Rightarrow$ (analytic) $\underline{[25]-2_{n}}: \quad n_{E}=l_{B}=l=k=k_{5}=m_{x}=0, l_{E}+l_{5}=1, \forall n_{B}, \forall m_{t} \Rightarrow$ (numeric).

$$
\gamma_{3}=\gamma_{3}\left(\partial_{t}, \vec{\nabla}, 0,0 ; 0,0, \vec{E} \cdot \vec{B}\right), \quad \gamma_{4}=\gamma_{4}\left(\partial_{t}, \vec{\nabla}, 0,0 ; E^{2}, 0,0\right)
$$

- In the present work, we relax some of the approximations made in [24, 25] and derive constitutive relations for the currents, up to third order in the gradient expansion.

$$
\begin{array}{rlrl}
n_{E} & +n_{B}+l_{E}+l_{B}+m_{t}+m_{x} \leq 3, \forall\left(l, l_{5}, k, k_{5}\right) & \Rightarrow \text { (analytic) } \\
\gamma_{1,4} & =\gamma_{1,4}\left(\partial_{t}, 0,0,0 ; E^{2}, 0,0\right), & \gamma_{2} & =\gamma_{2}(0,0,0, \vec{B} \cdot \vec{\nabla} ; 0,0,0), \\
\gamma_{3} & =\gamma_{3}\left(\partial_{t}, \vec{\nabla}, 0,0 ; 0,0, \vec{E} \cdot \vec{B}\right), & \gamma_{5,8,9} & =\gamma_{5,8,9}\left(\partial_{t}, 0,0,0 ; 0,0,0\right), \\
\gamma_{7,18} & =\gamma_{7,18}\left(\partial_{t}, 0,0,0 ; 0,0,0\right), & \gamma_{10,11,12} & =\gamma_{10,11,12}(0,0,0,0 ; 0,0,0) .
\end{array}
$$

- In the forthcoming paper [96], we will primarily focus on those TCFs associated with nonlinear terms at $\mathcal{O}\left(\epsilon^{1} \alpha^{1}\right)$.

$$
\begin{aligned}
\underline{[96]-1_{a}:}: l_{E} & =l_{B}=0, n_{E}+n_{B}=1, l+l_{5}=1, \forall\left(k, k_{5}\right), m_{t}+m_{x} \leq 3 \Rightarrow \text { (analytic) } \\
\underline{[96]-1_{n}}: \quad l_{E} & =l_{B}=0, n_{E}+n_{B}=1, l+l_{5}=1, \forall\left(k, k_{5}, m_{t}, m_{x}\right) \Rightarrow \text { (numeric) } \\
\gamma_{1,2} & =\gamma_{1,2}\left(\partial_{t}, \vec{\nabla}, \vec{E} \cdot \vec{\nabla}, \vec{B} \cdot \vec{\nabla} ; 0,0,0\right), \\
\gamma_{i} & =\gamma_{i}\left(\partial_{t}, \vec{\nabla}, 0,0 ; 0,0,0\right), \quad i=4,6-8,15,17,18 .
\end{aligned}
$$

In the next subsection, we summarise the main results of the present work, leaving all the technical details in the main text and appendix. 


\subsection{Main results}

This subsection is further split into three parts. The first one contains the general form of the currents. The second part focuses on the second order nonlinear transport and comparison with similar results obtained in the CKT. The third order terms constituting the bulk of our new results are presented towards the end of this subsection.

\subsubsection{General form of the currents}

A formal expression for the constitutive relations for the vector and axial currents is derived in section 4 , having the following form

$$
\begin{array}{ll}
J^{t}=\rho, & \vec{J}=-\mathcal{D}_{0} \vec{\nabla} \rho+\sigma_{e}^{0} \vec{E}+\sigma_{\chi}^{0} \mu_{5} \vec{B}+\delta \vec{J}, \\
J_{5}^{t}=\rho_{5}, & \overrightarrow{J_{5}}=-\mathcal{D}_{0} \vec{\nabla} \rho_{5}+\sigma_{\chi}^{0} \mu \vec{B}+\delta \vec{J}_{5},
\end{array}
$$

where $\rho, \rho_{5}$ are generic vector/axial charge densities and $\mu, \mu_{5}$ are corresponding chemical potentials. The external e/m fields $\vec{E}, \vec{B}$ are generic as well, that is none of the approximations introduced in $[24,25]$ is assumed. The lowest order TCs - charge diffusion constant $\mathcal{D}_{0}$, DC electrical conductivity $\sigma_{e}^{0}$ and DC CME/CSE conductivity $\sigma_{\chi}^{0}$ are $[24,111]$

$$
\mathcal{D}_{0}=\frac{1}{2}, \quad \sigma_{e}^{0}=1, \quad \sigma_{\chi}^{0}=12 \kappa,
$$

where from here on we set $\pi T=1$ for convenience. Proper powers of $\pi T$ for dimensionfull quantities could be easily recovered given their physical dimensions.

The $\sigma_{\chi}^{0}$-terms in $\vec{J}$ and $\vec{J}_{5}$ are standard CME and CSE, respectively, in agreement with "non-renormalisability" of CME $[95,109,110]$. The new element here, which we find important to emphasise, is that (2.11), (2.12) are exact, that is they are derived relaxing all the approximations undertaken in $[24,25,109,110]$. Nonlinearity of CME/CSE in external fields $\vec{E}$ and $\vec{B}$ is completely absorbed into the chemical potentials $\mu, \mu_{5}$.

The corrections $\delta \vec{J}$ and $\delta \vec{J}_{5}$ are formally defined in (A.7), which consist of higher derivative terms starting from the second order only. These terms are built from powers and derivatives of $\vec{E}, \vec{B}, \mu$ and $\mu_{5} . \quad \delta \vec{J}$ and $\delta \vec{J}_{5}$ are not known analytically, but could be worked out perturbatively. $\delta \vec{J}$ and $\delta \vec{J}_{5}$ introduce new effects, particularly additional contributions to the currents along the direction of $\vec{B}$ proportional to derivatives of the chemical potentials. These effects introduce very important modifications to the original CME/CSE. As will be clear later, external e/m fields make corrections to $\mathcal{D}_{0}$ and $\sigma_{e}^{0}$, and even generalise them into tensor-type TCs. While in principle an axial analogue of $\sigma_{e}^{0}$ (i.e., a term proportional to $\vec{E}$-term) in $\vec{J}_{5}$ is also possible, it does not appear in our calculations due to the probe limit [113].

We have mentioned earlier a discussion about vanishing equilibrium CME, which might appear in tension with (2.11). In principle, since $\mathrm{U}(1)_{A}$ is not a symmetry, axial gauge potential $\mathcal{A}_{\mu}$ itself could be regarded as another external field. Our calculations, however, are performed assuming vanishing $\mathcal{A}_{\mu}$. Had we introduced a non-vanishing constant background for the time component, $\mathcal{A}_{t} \neq 0$, CME conductivity $\sigma_{\chi}^{0}$ would be shifted

$$
\vec{J}_{\mathrm{CME}}=12 \kappa\left(\bar{\mu}_{5}-\mathcal{A}_{t}\right) \vec{B},
$$


due to a Chern-Simons contribution [24, 64]. In order to have CME vanish, it suffices to impose $\mathcal{A}_{t}=\bar{\mu}_{5}[46,64]$. In [46] it was indeed argued that the equality $\mathcal{A}_{t}=\bar{\mu}_{5}$ must be satisfied in equilibrium. While we do not have much to add to this discussion, we notice that a constant $\mathcal{A}_{t}$ does not lead to any new effect beyond the shift (2.14) in CME. This is because the bulk dynamics underlying our model is expressed entirely in terms of the vector and axial field strengths.

\subsubsection{Second order results: comparison with the CKT}

At second order, the results read

$$
\begin{aligned}
\delta \vec{J}= & \frac{1}{4} \sigma_{m}^{0}\left(\rho^{2}+\rho_{5}^{2}\right) \vec{\nabla} \times \vec{B}-\frac{1}{4} \mathcal{D}_{H}^{0} \vec{B} \times\left(\rho \vec{\nabla} \rho+\rho_{5} \vec{\nabla} \rho_{5}\right)-\frac{1}{2} \sigma_{a \chi H}^{0} \vec{E} \times \vec{\nabla} \rho_{5} \\
& -\tau_{e} \sigma_{e}^{0} \partial_{t} \vec{E}-\frac{1}{2} \sigma_{\chi H}^{0} \rho \vec{B} \times \vec{E}-\frac{1}{2} \tau_{\chi} \rho_{5} \partial_{t} \vec{B}+\tau_{D} \partial_{t} \vec{\nabla} \rho+\tau_{\bar{\chi}}\left(\partial_{t} \rho_{5}\right) \vec{B}+\mathcal{O}\left(\partial^{3}\right), \\
\delta \overrightarrow{J_{5}}= & \frac{1}{2} \sigma_{m}^{0} \rho \rho_{5} \vec{\nabla} \times \vec{B}-\frac{1}{4} \mathcal{D}_{H}^{0} \vec{B} \times\left(\rho \vec{\nabla} \rho_{5}+\rho_{5} \vec{\nabla} \rho\right)-\frac{1}{2} \sigma_{a \chi H}^{0} \vec{E} \times \vec{\nabla} \rho \\
& -\frac{1}{2} \sigma_{\chi H}^{0} \rho_{5} \vec{B} \times \vec{E}-\frac{1}{2} \tau_{\chi} \rho \partial_{t} \vec{B}+\tau_{D} \partial_{t} \vec{\nabla} \rho_{5}+\tau_{\bar{\chi}}\left(\partial_{t} \rho\right) \vec{B}+\mathcal{O}\left(\partial^{3}\right),
\end{aligned}
$$

where the TCs take the following values

$$
\begin{aligned}
& \sigma_{m}^{0}=72(2 \log 2-1) \kappa^{2}, \quad \mathcal{D}_{H}^{0}=72(3 \log 2-2) \kappa^{2}, \quad \sigma_{a \chi H}^{0}=6 \log 2 \kappa, \\
& \sigma_{\chi H}^{0}=72 \log 2 \kappa^{2}, \quad \tau_{e}=\frac{\log 2}{2}, \quad \tau_{\chi}=12 \log 2 \kappa, \\
& \tau_{D}=\frac{\pi}{8}, \quad \tau_{\bar{\chi}}=-\left(\frac{3}{2} \pi+3 \log 2\right) \kappa .
\end{aligned}
$$

The TCs in (2.15), (2.16) could be related to Taylor expansion of some $\gamma_{i}$ 's in (2.4), schematically indicated as follows

$$
\begin{aligned}
\sigma_{m}^{0} \in \gamma_{5}, \quad \mathcal{D}_{H}^{0} \in \gamma_{8}, \quad \sigma_{a \chi H}^{0} \in \gamma_{9}, \quad \sigma_{\chi H}^{0} \in \gamma_{11}, \quad \tau_{e} \in \gamma_{3}, \\
\tau_{\chi}, \tau_{\bar{\chi}} \in \gamma_{4}, \quad \tau_{D} \in \gamma_{1} .
\end{aligned}
$$

To the best of our knowledge, in a holographic model, $\sigma_{a \chi H}^{0}$ is computed here for the first time. The rest of the TCs in (2.15), (2.16) have been previously computed in [24, 25]. While in the constitutive relations these terms were already introduced in [24, 25], they did not contribute to dynamics (continuity equations) due to the static/homogeneous field approximations assumed in these earlier publications. For instance, to $\mathcal{O}\left(\epsilon^{0}\right)$ considered in [24], the currents do not contain any gradient terms, resulting in dynamical instability (linear growth of $\rho_{5}$ with time). Inclusion of the external e/m field fluctuations (i.e., $\delta \vec{E}$ and $\delta \vec{B}$ ) and associated gradient terms in (2.15), (2.16) regularizes the instability. Our results (2.15), (2.16) reveal new dynamical effects and thus are novel in a sense of a much broader range of applicability. Physical interpretation of the terms in (2.15), (2.16) and their dissipative properties are discussed below.

Our second order results could be compared with similar results obtained in CKT $[103,104]$. To this goal, we first put the currents on-shell eliminating $\partial_{t} \rho$ and $\partial_{t} \rho_{5}$ 
using the continuity equations (1.1). Second, we replace the densities by the corresponding chemical potentials. In the holographic model, the chemical potentials $\mu, \mu_{5}$ are computed analytically in the hydrodynamic limit. At second order in the gradient expansion,

$$
\begin{aligned}
\mu= & \frac{1}{2} \rho+\frac{1}{16}(\pi-2 \log 2) \vec{\nabla}^{2} \rho-\frac{3}{4}(\pi-2 \log 2) \kappa\left(\vec{B} \cdot \vec{\nabla} \rho_{5}\right)+18(1-2 \log 2) \kappa^{2} \rho B^{2} \\
& -\frac{1}{8}(\pi+2 \log 2)(\vec{\nabla} \cdot \vec{E})+\mathcal{O}\left(\partial^{3}\right), \\
\mu_{5}= & \frac{1}{2} \rho_{5}+\frac{1}{16}(\pi-2 \log 2) \vec{\nabla}^{2} \rho_{5}-\frac{3}{4}(\pi-2 \log 2) \kappa(\vec{B} \cdot \vec{\nabla} \rho)+18(1-2 \log 2) \kappa^{2} \rho_{5} B^{2} \\
& +\frac{3}{2}(\pi-2 \log 2) \kappa(\vec{E} \cdot \vec{B})+\mathcal{O}\left(\partial^{3}\right) .
\end{aligned}
$$

Eventually, the on-shell currents are

$$
\begin{aligned}
\vec{J}^{\text {on }- \text { shell }}= & \sigma_{\chi}^{0} \mu_{5} \vec{B}-\tau_{\chi} \mu_{5} \partial_{t} \vec{B}+\sigma_{e}^{0}(\vec{E}-\vec{\nabla} \mu)-\tau_{e} \sigma_{e}^{0} \partial_{t} \vec{E}+\sigma_{\chi H}^{0} \mu \vec{E} \times \vec{B} \\
& -\mathcal{D}_{H}^{0} \vec{B} \times\left(\mu \vec{\nabla} \mu+\mu_{5} \vec{\nabla} \mu_{5}\right)-\sigma_{a \chi H}^{0} \vec{E} \times \vec{\nabla} \mu_{5}+\sigma_{m}^{0}\left(\mu^{2}+\mu_{5}^{2}\right) \vec{\nabla} \times \vec{B}+\mathcal{O}\left(\partial^{3}\right), \\
\vec{J}_{5}^{\text {on }- \text { shell }}= & \sigma_{\chi}^{0} \mu \vec{B}-\tau_{\chi} \mu \partial_{t} \vec{B}-\sigma_{e}^{0} \vec{\nabla} \mu_{5}+\sigma_{\chi H}^{0} \mu_{5} \vec{E} \times \vec{B}-\mathcal{D}_{H}^{0} \vec{B} \times\left(\mu \vec{\nabla} \mu_{5}+\mu_{5} \vec{\nabla} \mu\right) \\
& -\sigma_{a \chi H}^{0} \vec{E} \times \vec{\nabla} \mu+2 \sigma_{m}^{0} \mu \mu_{5} \vec{\nabla} \times \vec{B}+\mathcal{O}\left(\partial^{3}\right) .
\end{aligned}
$$

Now lets discuss the physics of each term in (2.23), (2.24), primarily focusing on $\vec{J}^{\text {on-shell }}$. The first term in (2.23) is CME. The next one introduces relaxation into CME induced by time variation of the magnetic field, with $\tau_{\chi}$ being a relaxation time originally computed in [24]. $\tau_{\chi}$ was recently re-examined numerically in [114] within a quite similar holographic model but beyond probe limit. The third and fourth terms are just the classic Ohm's and diffusion currents accompanied by another relaxation effect associated with time varying electric field. The corresponding relaxation time $\tau_{e}$ was originally computed in [115]. Note that in $(2.15),(2.16)$ there are two additional relaxation time terms. The first one with $\tau_{D}$ enters the diffusion current [111]. Finally, $\tau_{\bar{\chi}}$ is yet another relaxation time associated with generalised CME. Note the difference between $\tau_{\chi}$ and $\tau_{\bar{\chi}}$ : while the former is a TC responding to time varying external magnetic field, the latter is related to relaxation of the axial charge density. In (2.23), (2.24) both terms appear as $\mathcal{O}\left(\partial^{3}\right)$.

$\sigma_{\chi}^{0}$ and $\tau_{\bar{\chi}}$ are the first two coefficients in the gradient expansion of a resummed TCF $\sigma_{\bar{\chi}}[96]$. Instead of a full resummation, which is a complicated numerical problem, one could use the relaxation time $\tau_{\bar{\chi}}$ in order to build a causal model for $\sigma_{\bar{\chi}}$ in a spirit of MIS. In this sense, the relaxation times, such as $\tau_{\chi}$ and $\tau_{\bar{\chi}}$, are of special importance.

The $\vec{E} \times \vec{B}$-term in (2.23), (2.24) looks very similar to the usual Hall effect, which is, however, absent in our holographic model because of the probe limit. The term that we do find is induced by the chiral anomaly. To distinguish it from the normal Hall effect, it is referred to as chiral Hall effect [116] with $\sigma_{\chi H}^{0}$ being its TC. Notice that $\sigma_{\chi H}^{0} \propto \kappa^{2}$. Contrary to purely anomaly-induced effects, which are normally odd in $\kappa$, the terms even in $\kappa$ appear as anomaly-induced corrections to normal transports [107]. The $\mathcal{D}_{H}^{0}$-term generates current perpendicular to both the magnetic field and gradients of chemical potentials. In [104] this effect was called Hall diffusion. This term can be 
regarded as an example (we will expand on this below) in which the diffusion constant is turned into a non-trivial diffusion tensor depending on the magnetic field.

The $\sigma_{a \chi H^{-}}^{0}$-term in (2.23) induces flow perpendicular to both the electric field and gradient of the axial chemical potential. It was referred to as anomalous chiral Hall effect in $[104]{ }^{7}$

Finally, the last term in (2.23) corresponds to another anomaly-induced correction to a normal current. Normal transport due to rotor of magnetic field was first analysed in [111]. At second order in the gradient expansion under discussion now, the "normal" transport coefficient was found to be identically zero. Thus, the entire effect at this order arises from the anomaly alone.

Dissipative nature of each term entering (2.23), (2.24) is of interest. While we do not intend to dwell in this question here, we note in passing that the TCs $\sigma_{\chi}^{0}, \tau_{e}, \sigma_{\chi H}^{0}, \mathcal{D}_{H}^{0}$, $\sigma_{m}^{0}$, and $\tau_{D}$ are all time reversal $\mathcal{T}$-even and thus non-dissipative. The remaining terms in $(2.23),(2.24)$ are dissipative.

Starting from CSE, the various terms in $\vec{J}_{5}^{\text {on-shell }}$ could be simply understood as axial analogues of those in $\vec{J}^{\text {on-shell }}$.

For the sake of a more detailed comparison of our results with parallel ones in CKT, we quote here the expression for the vector current as appears in [104]

$$
\begin{aligned}
\vec{J}_{\mathrm{CKT}}= & \frac{1}{2 \pi^{2}} \mu_{5} \vec{B}-\frac{\tau \mu_{5}}{6 \pi^{2}} \partial_{t} \vec{B}+\sigma_{e}^{\mathrm{CKT}}(\vec{E}-\vec{\nabla} \mu)-\tau \sigma_{e}^{\mathrm{CKT}} \partial_{t} \vec{E}+\sigma_{H}^{\mathrm{CKT}} \mu \vec{E} \times \vec{B} \\
& -\mathcal{D}_{H}^{\mathrm{CKT}}\left(\mu \vec{\nabla} \mu+\mu_{5} \vec{\nabla} \mu_{5}\right) \times \vec{B}-\sigma_{a \chi}^{\mathrm{CKT}} \vec{E} \times \vec{\nabla} \mu_{5}-\mathcal{D}_{\chi}^{\mathrm{CKT}} \mu \mu_{5} \vec{\nabla} \mu_{5},
\end{aligned}
$$

where

$$
\begin{aligned}
& \sigma_{e}^{\mathrm{CKT}}=\frac{\tau}{9 \pi^{2}}\left[1+3\left(\mu^{2}+\mu_{5}^{2}\right)\right], \\
& \sigma_{H}^{\mathrm{CKT}}=\frac{\tau^{2}}{3 \pi^{2}}, \\
& \mathcal{D}_{H}^{\mathrm{CKT}}=\frac{\tau^{2}}{3 \pi^{2}}, \\
& \sigma_{a \chi H}^{\mathrm{CKT}}=\frac{\tau}{6 \pi^{2}}, \\
& \mathcal{D}_{\chi}^{\mathrm{CKT}}=\frac{2 \tau}{3 \pi^{2}} \text {. }
\end{aligned}
$$

Here $\tau$ is a parameter of dimension of time introduced in relaxation time approximation (RTA) of CKT. Confronting with (2.23) we notice absence of $\vec{\nabla} \mu_{5}$ term in $\vec{J}$ on-shell. Similarly, there are no terms proportional to $\vec{\nabla} \mu, \vec{E}, \partial_{t} \vec{E}$ in $\vec{J}_{5}{ }^{\text {on-shell }}$. All these terms are expected to arise beyond the probe limit. On the other hand, the magnetic conductivity term $\vec{\nabla} \times \vec{B}$ is missing in (2.25). All the remaining terms appear in perfect agreement, at least as far as general structures are concerned.

Because in principle the two models describe two different regimes (strong vs weak coupling), the transport coefficients are not expected to agree. It is nevertheless instructive to pursue such a comparison. For this goal, we need to fix the parameter $\tau$ of the CKT. Obviously, there is no unique way to fix $\tau$. We chose to set CME as a benchmark. That is, we equate the CME conductivities and the associated relaxation times in two models. This results in

$$
\kappa=\frac{1}{24 \pi^{2}}, \quad \tau=3 \log 2 .
$$

\footnotetext{
${ }^{7}$ Indeed, the $\tau_{\chi^{-}}, \sigma_{\chi H^{-}}^{0}$-terms in $(2.23)$ could be reorganised as $-\sigma_{a \chi H}^{0}\left(\vec{E} \times \vec{\nabla} \mu_{5}+\mu_{5} \partial_{t} \vec{B}\right)-\sigma_{a \chi H}^{0} \mu_{5} \partial_{t} \vec{B}$. More precisely it is $\left(\vec{E} \times \vec{\nabla} \mu_{5}+\partial_{t} \vec{B}\right)$-term that was called anomalous chiral Hall effect in [104].
} 
Then, the transport coefficients in (2.23) are compared to those in (2.25)

$$
\begin{aligned}
& \sigma_{e}^{0} / \sigma_{e}^{\mathrm{CKT}} \sim \mathcal{O}\left(10^{2}\right), \quad \tau_{e} / \tau^{C K T} \sim \mathcal{O}\left(10^{-1}\right), \quad \sigma_{\chi H}^{0} / \sigma_{H}^{\mathrm{CKT}} \sim \mathcal{O}\left(10^{-3}\right), \\
& \mathcal{D}_{H}^{0} / \mathcal{D}_{H}^{\mathrm{CKT}} \sim \mathcal{O}\left(10^{-3}\right), \quad \sigma_{a \chi}^{0} / \sigma_{a \chi}^{\mathrm{CKT}} \sim \mathcal{O}(1) \text {. }
\end{aligned}
$$

While some of the coefficients came out to be of the same order, the electrical conductivity $\sigma_{e}^{\mathrm{CKT}}$ in CKT is strongly suppressed (by order $10^{-2}$ ) compared to the holographic model. On the other hand, the anomaly-induced coefficients $\sigma_{\chi H}^{0}$ and $\mathcal{D}_{H}^{0}$ are highly suppressed (by order $10^{-3}$ ) in holography. ${ }^{8}$

A complimentary way of looking at $(2.11),(2.12),(2.15),(2.16)$ is by separately collecting terms proportional to $\vec{\nabla} \rho$ and $\vec{\nabla} \rho_{5}$. All these terms constitute a diffusive current, which to the lowest order in the gradients is

$$
J_{\text {diff }}^{i}=-\mathcal{D}_{i j}^{0} \nabla_{j} \rho-\left(\mathcal{D}_{\chi}^{0}\right)_{i j} \nabla_{j} \rho_{5},
$$

where

$$
\mathcal{D}_{i j}^{0}=\frac{1}{4}\left(4 \mathcal{D}_{0} \delta_{i j}+\mathcal{D}_{H}^{0} \epsilon_{i k j} B_{k} \rho\right), \quad\left(\mathcal{D}_{\chi}^{0}\right)_{i j}=\frac{1}{4}\left(2 \sigma_{a \chi}^{0} \epsilon_{i k j} E_{k}+\mathcal{D}_{H}^{0} \epsilon_{i k j} B_{k} \rho_{5}\right) .
$$

Much like in MHD, the diffusion constants are turned into tensors, which in fact depend non-linearly on the external e/m fields $E$ and $B$. Furthermore, when higher order gradients are resummed, these diffusion tensors become momenta dependent tensor functions [96].

\subsubsection{The third order results and collective excitations}

Third order corrections in $\delta \vec{J}$ and $\delta \vec{J}_{5}$ contain a few dozens of new terms with corresponding TCs, all of which are computed analytically (see section 4 for more details). Since the complete set of the results for $\delta \vec{J}^{[3]}$ and $\delta \vec{J}_{5}^{[3]}$ is very large, in this Summary section we focus only on the most interesting terms, the ones which are linear in $\rho, \rho_{5}$, while the remaining nonlinear in $\rho, \rho_{5}$ corrections are flashed in section 4 .

Denoted as $\delta \vec{J}^{[3] l}$ and $\delta \vec{J}_{5}^{[3] l}$, the linear in $\rho, \rho_{5}$ terms at third order are

$$
\begin{aligned}
\delta \vec{J}^{[3] l}= & \tau_{1} \partial_{t}^{2}(\vec{\nabla} \rho)+\tau_{2} \vec{\nabla}^{2}(\vec{\nabla} \rho)+\tau_{3} \partial_{t}^{2} \vec{E}+\tau_{4} \vec{\nabla}^{2} \vec{E}+\tau_{5} \partial_{t}(\vec{\nabla} \times \vec{B})+\tau_{6}\left(\partial_{t}^{2} \rho_{5}\right) \vec{B} \\
& +\tau_{7} \partial_{t} \rho_{5} \partial_{t} \vec{B}+\tau_{8} \rho_{5} \partial_{t}^{2} \vec{B}+\tau_{9}\left(\vec{\nabla}^{2} \rho_{5}\right) \vec{B}+\tau_{10} \rho_{5} \vec{\nabla}^{2} \vec{B}+\tau_{11}\left(\vec{\nabla} \rho_{5} \cdot \vec{\nabla}\right) \vec{B} \\
& +\tau_{12}(\vec{B} \cdot \vec{\nabla}) \vec{\nabla} \rho_{5}+\widetilde{\tau}_{12} \vec{\nabla}(\vec{B} \cdot \vec{\nabla}) \rho_{5}+\frac{\tau_{13} \vec{\nabla}\left(\rho B^{2}\right)}{\tau_{14} \vec{E} \times(\vec{E} \times \vec{\nabla} \rho)} \\
& +\tau_{15} \partial_{t}\left(\vec{E} \times \vec{\nabla} \rho_{5}\right)+\tau_{16} \partial_{t} \vec{E} \times \vec{\nabla} \rho_{5}+\tau_{17} \rho \vec{E} \times \partial_{t} \vec{B}+\tau_{18} \partial_{t}(\rho \vec{B} \times \vec{E}) \\
& +\tau_{19} \rho_{5} \vec{E} \times(\vec{E} \times \vec{B})+\tau_{20} \vec{E} \times \partial_{t}(\rho \vec{B}),
\end{aligned}
$$

\footnotetext{
${ }^{8}$ In the presented comparison, we have expressed the constitutive relations in terms of the chemical potentials, so to have them in the same form as in [104]. We could have done oppositely and that is to compare the results expressed in terms of the charge densities. To this goal we could use the relation between the charge densities and the chemical potentials of [104], which remarkably are quite similar to (2.21), (2.22). While on both sides of the comparison the TCs get modified, we have checked that the ratios (2.28) remain intact.
} 


$$
\begin{aligned}
\delta \vec{J}_{5}^{[3] l}= & \tau_{1} \partial_{t}^{2}\left(\vec{\nabla} \rho_{5}\right)+\tau_{2} \vec{\nabla}^{2}\left(\vec{\nabla} \rho_{5}\right)+\tau_{6}\left(\partial_{t}^{2} \rho\right) \vec{B}+\tau_{7} \partial_{t} \rho \partial_{t} \vec{B}+\tau_{8} \rho \partial_{t}^{2} \vec{B}+\tau_{9}\left(\vec{\nabla}^{2} \rho\right) \vec{B} \\
& +\tau_{10} \rho \vec{\nabla}^{2} \vec{B}+\tau_{11}(\vec{\nabla} \rho \cdot \vec{\nabla}) \vec{B}+\tau_{12}(\vec{B} \cdot \vec{\nabla}) \vec{\nabla} \rho+\widetilde{\tau}_{12} \vec{\nabla}(\vec{B} \cdot \vec{\nabla}) \rho \\
& +\frac{\tau_{13} \vec{\nabla}\left(\rho_{5} B^{2}\right)}{\tau_{14} \vec{E} \times\left(\vec{E} \times \vec{\nabla} \rho_{5}\right)}+\tau_{15} \partial_{t}(\vec{E} \times \vec{\nabla} \rho)+\tau_{16} \partial_{t} \vec{E} \times \vec{\nabla} \rho \\
& +\tau_{17} \rho_{5} \vec{E} \times \partial_{t} \vec{B}+\tau_{18} \partial_{t}\left(\rho_{5} \vec{B} \times \vec{E}\right)+\tau_{19} \rho \vec{E} \times(\vec{E} \times \vec{B})+\tau_{20} \vec{E} \times \partial_{t}\left(\rho_{5} \vec{B}\right) \\
& +\tau_{21} \vec{E} \times(\vec{\nabla} \times \vec{B})+\tau_{22} \vec{\nabla}(\vec{B} \cdot \vec{E}),
\end{aligned}
$$

where

$$
\widetilde{\tau}_{12}=\tau_{12}+\tau_{10}-\tau_{9}
$$

In (2.31), (2.32) we have made use of the Bianchi identity (1.4) and eliminated $\vec{\nabla} \times \vec{E}$. The values of TCs $\tau_{1-22}$ are collected in appendix A, see (A.28)-(A.50). Apart from the $\tau_{3}, \tau_{4}, \tau_{5}, \tau_{21}, \tau_{22}$ terms, one can obtain $\delta \vec{J}_{5}^{[3] l}$ from $\delta \vec{J}^{[3] l}$ via exchange of $\rho$ and $\rho_{5}$. It is important to give physical interpretation for $\tau_{i}$ in (2.31), (2.32).

The TCs $\tau_{1-5}$ represent the second order gradient expansion of the charge diffusion function $\mathcal{D}$, electric and magnetic conductivity functions $\sigma_{e}, \sigma_{m}$, and were first computed in [111] by employing weak field approximation. The $\tau_{8}, \tau_{10}$-terms are second order gradient expansion of CME conductivity $\sigma_{\chi}$ [24]. The $\tau_{19}$-term was first obtained in [24] for constant electromagnetic fields, which, once expanded, contains nonlinear corrections to the original $\mathrm{CME} / \mathrm{CSE}$.

The underlined terms $\tau_{13}, \tau_{14}$ include anomaly-induced $B^{2}-, E^{2}$-corrections to the charge diffusion constant $\mathcal{D}_{0}$ :

$$
\mathcal{D}_{0}=\frac{1}{2}-18(2 \log 2-1) \kappa^{2} B^{2}-\frac{3}{4} \pi^{2} \kappa^{2} E^{2}+\cdots .
$$

We note that both corrections are negative, see (2.34). $E^{2}$-correction is new whereas $B^{2}$ correction was first calculated in [25]. Obviously, there will be higher powers in $E^{2}, B^{2}$ corrections to $\mathcal{D}_{0}$. In the forthcoming publication [117], we will compute the charge diffusion constant, as a function of constant $\mathrm{e} / \mathrm{m}$ fields relaxing the weak field approximation.

The transport coefficients $\tau_{6}, \tau_{7}, \tau_{9}$ are due to spacetime inhomogeneity of $\rho, \rho_{5} . \tau_{6}, \tau_{9}$ correspond to second order expansion of the generalised CME/CSE conductivity function $\sigma_{\bar{\chi}}$ to be computed in the forthcoming paper [96].

The terms $\tau_{11}, \tau_{12}, \widetilde{\tau}_{12}$ represent mixing effect between magnetic field and spatial gradients of $\rho, \rho_{5}$. They were first considered in [25]. The TCs $\tau_{15-18}, \tau_{20}$ have similar structure as the Hall diffusion and Hall effect, but the former are induced by time-varying densities and electromagnetic fields. Thus, the $\tau_{15-18}, \tau_{20}$-terms are relaxation times corrections to the Hall diffusion and Hall effect.

The $\tau_{21}, \tau_{22}$-terms are due to spatial inhomogeneity of electromagnetic fields. Vector analogs of $\tau_{21}, \tau_{22}$ will emerge as nonlinear in $\rho, \rho_{5}$ terms, see $\tau_{30}, \tau_{34}$-terms in (4.6).

Via the criterion for dissipative/non-dissipative transports based on $\mathcal{T}$-symmetry arguments, the TCs $\tau_{6-12}, \widetilde{\tau}_{12}, \tau_{15}, \tau_{16}, \tau_{19}, \tau_{21}$ and $\tau_{22}$ are $\mathcal{T}$-even and thus correspond to non-dissipative TCs, while the rest of the terms are dissipative.

The third order gradient corrections (2.31), (2.32) contribute to various collective excitations of the holographic chiral medium, particularly they modify the dispersion relation 
of CMW [95]. For constant electromagnetic fields,

$$
\begin{aligned}
\omega= & \pm\left[1-36(2 \log 2-1) \kappa^{2} \mathbf{B}^{2}-\frac{3 \pi^{2}}{2} \kappa^{2} \mathbf{E}^{2}\right] 6 \kappa(\vec{q} \cdot \overrightarrow{\mathbf{B}}) \pm 9 \pi^{2}(\overrightarrow{\mathbf{E}} \cdot \overrightarrow{\mathbf{B}}) \kappa^{3}(\vec{q} \cdot \overrightarrow{\mathbf{E}}) \\
& +(36 \log 2) \kappa^{2}(\vec{q} \cdot \overrightarrow{\mathbf{S}})-\left[\frac{1}{2}+18(1-2 \log 2) \kappa^{2} \mathbf{B}^{2}-\frac{3 \pi^{2}}{4} \kappa^{2} \mathbf{E}^{2}\right] i q^{2} \\
& \pm \frac{9}{2} \log 2 \kappa(\vec{q} \cdot \overrightarrow{\mathbf{B}}) q^{2}-\frac{i}{8} q^{4} \log 2-i \frac{3}{4} \pi^{2} \kappa^{2}(\vec{q} \cdot \overrightarrow{\mathbf{E}})^{2}+i(36 \log 2) \kappa^{2}(\vec{q} \cdot \overrightarrow{\mathbf{B}})^{2}+\cdots
\end{aligned}
$$

When $\overrightarrow{\mathbf{E}}=0$, the dispersion relation (2.35) reduces to the one obtained in [25]. The first term $(\sim \vec{q} \cdot \overrightarrow{\mathbf{B}})$ in $(2.35)$ represents nonlinear corrections to the speed of CMW, which are negative making the wave to propagate slower. The second term $(\sim \vec{q} \cdot \overrightarrow{\mathbf{E}})$ in $(2.35)$ corresponds to a wave mode propagating along the electric field. It is called density wave [33] or chiral electric wave [116]. Since the chiral electric separation effect vanishes in the probe limit, here this effect is mimicked by the second term in (2.35) which is induced by the chiral anomaly as a nonlinear correction. Its presence is conditional to $\overrightarrow{\mathbf{E}}$ not being orthogonal to $\overrightarrow{\mathbf{B}}$. We find the third term $(\sim \vec{q} \cdot \overrightarrow{\mathbf{S}})$ of special interest because it corresponds to a new phenomenon. It corresponds to a wave propagating along the direction of the energy flux $\overrightarrow{\mathbf{S}}=\overrightarrow{\mathbf{E}} \times \overrightarrow{\mathbf{B}}$, which can be referred to as chiral Hall density wave (CHDW). The remaining terms in (2.35) are decay rates of various wave modes.

The rest of this paper is structured as follows. Section 3 is about the holographic model. Section 4 is devoted to the main part of our study. Section 5 contains some closing remarks. Appendix A collects more technical details.

\section{Holographic setup: $\mathrm{U}(1)_{V} \times \mathrm{U}(1)_{A}$}

The holographic model is Maxwell-Chern-Simons theory in the Schwarzschild- $A d S_{5}$. The bulk action is

$$
S=\int d^{5} x \sqrt{-g} \mathcal{L}+S_{\text {c.t. }}
$$

where

$$
\begin{aligned}
\mathcal{L}= & -\frac{1}{4}\left(F^{V}\right)_{M N}\left(F^{V}\right)^{M N}-\frac{1}{4}\left(F^{a}\right)_{M N}\left(F^{a}\right)^{M N}+\frac{\kappa \epsilon^{M N P Q R}}{2 \sqrt{-g}} \\
& \times\left[3 A_{M}\left(F^{V}\right)_{N P}\left(F^{V}\right)_{Q R}+A_{M}\left(F^{a}\right)_{N P}\left(F^{a}\right)_{Q R}\right]
\end{aligned}
$$

and the counter-term action $S_{\text {c.t. }}$ is

$$
S_{\text {c.t. }}=\frac{1}{4} \log r \int d^{4} x \sqrt{-\gamma}\left[\left(F^{V}\right)_{\mu \nu}\left(F^{V}\right)^{\mu \nu}+\left(F^{a}\right)_{\mu \nu}\left(F^{a}\right)^{\mu \nu}\right] .
$$

The gauge Chern-Simons terms $(\sim \kappa)$ in the bulk action mimic the chiral anomaly of the boundary field theory. Note $\epsilon^{M N P Q R}$ is the Levi-Civita symbol with the convention $\epsilon^{r t x y z}=+1$, while the Levi-Civita tensor is $\epsilon^{M N P Q R} / \sqrt{-g}$. The counter-term action (3.3) is specified based on minimal subtraction, which excludes finite contribution to the boundary currents from the counter-term. 
In the ingoing Eddington-Finkelstein coordinate, the Schwarzschild- $A d S_{5}$ is

$$
d s^{2}=g_{M N} d x^{M} d x^{N}=2 d t d r-r^{2} f(r) d t^{2}+r^{2} \delta_{i j} d x^{i} d x^{j},
$$

where $f(r)=1-1 / r^{4}$. Thus, the Hawking temperature (identified as temperature of the boundary theory) is normalised to $\pi T=1$. On the hypersurface $\Sigma$ of constant $r$, the induced metric $\gamma_{\mu \nu}$ is

$$
\left.d s^{2}\right|_{\Sigma}=\gamma_{\mu \nu} d x^{\mu} d x^{\nu}=-r^{2} f(r) d t^{2}+r^{2} \delta_{i j} d x^{i} d x^{j} .
$$

It is convenient to split the bulk equations into dynamical and constraint components,

$$
\begin{array}{ll}
\text { dynamical equations : } & \mathrm{EV}^{\mu}=\mathrm{EA}^{\mu}=0, \\
\text { constraint equations : } & \mathrm{EV}^{r}=\mathrm{EA}^{r}=0,
\end{array}
$$

where

$$
\begin{aligned}
& \mathrm{EV}^{M} \equiv \nabla_{N}\left(F^{V}\right)^{N M}+\frac{3 \kappa \epsilon^{M N P Q R}}{\sqrt{-g}}\left(F^{a}\right)_{N P}\left(F^{V}\right)_{Q R}, \\
& \mathrm{EA}^{M} \equiv \nabla_{N}\left(F^{a}\right)^{N M}+\frac{3 \kappa \epsilon^{M N P Q R}}{2 \sqrt{-g}}\left[\left(F^{V}\right)_{N P}\left(F^{V}\right)_{Q R}+\left(F^{a}\right)_{N P}\left(F^{a}\right)_{Q R}\right] .
\end{aligned}
$$

The boundary currents are defined as

$$
J^{\mu} \equiv \lim _{r \rightarrow \infty} \frac{\delta S}{\delta V_{\mu}}, \quad J_{5}^{\mu} \equiv \lim _{r \rightarrow \infty} \frac{\delta S}{\delta A_{\mu}},
$$

which, in terms of the bulk fields, are

$$
\begin{aligned}
& J^{\mu}=\lim _{r \rightarrow \infty} \sqrt{-\gamma}\left\{\left(F^{V}\right)^{\mu M} n_{M}+\frac{6 \kappa \epsilon^{M \mu N Q R}}{\sqrt{-g}} n_{M} A_{N}\left(F^{V}\right)_{Q R}-\widetilde{\nabla}_{\nu}\left(F^{V}\right)^{\nu \mu} \log r\right\}, \\
& J_{5}^{\mu}=\lim _{r \rightarrow \infty} \sqrt{-\gamma}\left\{\left(F^{a}\right)^{\mu M} n_{M}+\frac{2 \kappa \epsilon^{M \mu N Q R}}{\sqrt{-g}} n_{M} A_{N}\left(F^{a}\right)_{Q R}-\widetilde{\nabla}_{\nu}\left(F^{a}\right)^{\nu \mu} \log r\right\},
\end{aligned}
$$

where $n_{M}$ is the outpointing unit normal vector with respect to the slice $\Sigma$, and $\widetilde{\nabla}$ is compatible with the induced metric $\gamma_{\mu \nu}$.

The radial gauge $V_{r}=A_{r}=0$ will be assumed throughout this work. As a result, in order to determine the boundary currents (3.11) it is sufficient to solve dynamical equations (3.6) only, leaving the constraints aside. Indeed, the constraint equations (3.7) give rise to continuity equations (1.1)

$$
\partial_{\mu} J^{\mu}=0, \quad \partial_{\mu} J_{5}^{\mu}=12 \kappa \vec{E} \cdot \vec{B} .
$$

In this way, the currents' constitutive relations to be derived below are off-shell.

Practically, it is more instructive to relate the currents (3.11) to the coefficients of near boundary asymptotic expansion of the bulk gauge fields. Near $r=\infty$,

$$
V_{\mu}=\mathcal{V}_{\mu}+\frac{V_{\mu}^{(1)}}{r}+\frac{V_{\mu}^{(2)}}{r^{2}}-\frac{2 V_{\mu}^{\mathrm{L}}}{r^{2}} \log r+\mathcal{O}\left(\frac{\log r}{r^{3}}\right), \quad A_{\mu}=\frac{A_{\mu}^{(2)}}{r^{2}}+\mathcal{O}\left(\frac{\log r}{r^{3}}\right)
$$


where

$$
V_{\mu}^{(1)}=\mathcal{F}_{t \mu}^{V}, \quad 4 V_{\mu}^{\mathrm{L}}=\partial^{\nu} \mathcal{F}_{\mu \nu}^{V} .
$$

A possible constant term for $A_{\mu}$ in (3.13) has been set to zero, in accordance with the fact that no axial external fields is assumed to be present in the current study. $\mathcal{V}_{\mu}$ is the gauge potential of external electromagnetic fields $\vec{E}$ and $\vec{B}$,

$$
E_{i}=\mathcal{F}_{i t}^{V}=\partial_{i} \mathcal{V}_{t}-\partial_{t} \mathcal{V}_{i}, \quad B_{i}=\frac{1}{2} \epsilon_{i j k} \mathcal{F}_{j k}^{V}=\epsilon_{i j k} \partial_{j} \mathcal{V}_{k}
$$

Dynamical equations (3.6) are sufficient to derive (3.13), (3.14), where the near-boundary data $V_{\mu}^{(2)}$ and $A_{\mu}^{(2)}$ have to be determined by completely solving (3.6) from the horizon to the boundary. The currents (3.11) become

$$
J^{\mu}=\eta^{\mu \nu}\left(2 V_{\nu}^{(2)}+2 V_{\nu}^{\mathrm{L}}+\eta^{\sigma t} \partial_{\sigma} \mathcal{F}_{t \nu}^{V}\right), \quad J_{5}^{\mu}=\eta^{\mu \nu} 2 A_{\nu}^{(2)} .
$$

As the remainder of this section, we outline the strategy for deriving the constitutive relations for $J^{\mu}$ and $J_{5}^{\mu}$. To this end, we turn on finite vector/axial charge densities for the dual field theory, which are also exposed to external electromagnetic fields. Holographically, the charge densities and external fields are encoded in asymptotic behaviors of the bulk gauge fields. In the bulk, we will solve the dynamical equations (3.6) assuming the charge densities and external fields as given, but without specifying them explicitly.

Following [111] we start with the most general static and homogeneous profiles for the bulk gauge fields satisfying the dynamical equations (3.6),

$$
V_{\mu}=\mathcal{V}_{\mu}-\frac{\rho}{2 r^{2}} \delta_{\mu t}, \quad A_{\mu}=-\frac{\rho_{5}}{2 r^{2}} \delta_{\mu t},
$$

where $\mathcal{V}_{\mu}, \rho, \rho_{5}$ are all constants for the moment. Regularity at $r=1$ has been used to fix one integration constant for each $V_{i}$ and $A_{i}$. As explained below (3.14), the constant term in $A_{\mu}$ is set to zero. Through (3.16), the boundary currents are

$$
J^{t}=\rho, \quad J^{i}=0 ; \quad J_{5}^{t}=\rho_{5}, \quad J_{5}^{i}=0 .
$$

Hence, $\rho$ and $\rho_{5}$ are identified as the vector/axial charge densities.

Next, following the idea of fluid/gravity correspondence [118], we promote $\mathcal{V}_{\mu}, \rho, \rho_{5}$ into arbitrary functions of the boundary coordinates

$$
\mathcal{V}_{\mu} \rightarrow \mathcal{V}_{\mu}\left(x_{\alpha}\right), \quad \rho \rightarrow \rho\left(x_{\alpha}\right), \quad \rho_{5} \rightarrow \rho_{5}\left(x_{\alpha}\right) .
$$

As a result, (3.17) ceases to solve the dynamical equations (3.6). To have them satisfied, suitable corrections in $V_{\mu}$ and $A_{\mu}$ have to be introduced:

$$
V_{\mu}\left(r, x_{\alpha}\right)=\mathcal{V}_{\mu}\left(x_{\alpha}\right)-\frac{\rho\left(x_{\alpha}\right)}{2 r^{2}} \delta_{\mu t}+\mathbb{V}_{\mu}\left(r, x_{\alpha}\right), \quad A_{\mu}\left(r, x_{\alpha}\right)=-\frac{\rho_{5}\left(x_{\alpha}\right)}{2 r^{2}} \delta_{\mu t}+\mathbb{A}_{\mu}\left(r, x_{\alpha}\right),
$$

where $\mathbb{V}_{\mu}, \mathbb{A}_{\mu}$ will be determined by solving (3.6). Appropriate boundary conditions are classified into three types. First, $\mathbb{V}_{\mu}$ and $\mathbb{A}_{\mu}$ are regular over the domain $r \in[1, \infty)$. Second, at the conformal boundary $r=\infty$, we require

$$
\mathbb{V}_{\mu} \rightarrow 0, \quad \mathbb{A}_{\mu} \rightarrow 0 \quad \text { as } \quad r \rightarrow \infty
$$


which amounts to fixing external gauge potentials to be $\mathcal{V}_{\mu}$ and zero (for the axial fields). Additional integration constants will be fixed by the Landau frame convention for the currents,

$$
J^{t}=\rho\left(x_{\alpha}\right), \quad J_{5}^{t}=\rho_{5}\left(x_{\alpha}\right) .
$$

The Landau frame convention corresponds to a residual gauge fixing for the bulk fields.

The vector/axial chemical potentials are defined as

$$
\begin{gathered}
\mu=V_{t}(r=\infty)-V_{t}(r=1)=\frac{1}{2} \rho-\mathbb{V}_{t}(r=1), \\
\mu_{5}=A_{t}(r=\infty)-A_{t}(r=1)=\frac{1}{2} \rho_{5}-\mathbb{A}_{t}(r=1) .
\end{gathered}
$$

Generically, $\mu, \mu_{5}$ are nonlinear functionals of densities and external fields.

In terms of $\mathbb{V}_{\mu}$ and $\mathbb{A}_{\mu}$, the dynamical equations (3.6) are

$$
\begin{aligned}
0= & r^{3} \partial_{r}^{2} \mathbb{V}_{t}+3 r^{2} \partial_{r} \mathbb{V}_{t}+r \partial_{r} \partial_{k} \mathbb{V}_{k}+12 \kappa \epsilon^{i j k}\left[\partial_{r} \mathbb{A}_{i}\left(\partial_{j} \mathcal{V}_{k}+\partial_{j} \mathbb{V}_{k}\right)+\partial_{r} \mathbb{V}_{i} \partial_{j} \mathbb{A}_{k}\right], \\
0= & \left(r^{5}-r\right) \partial_{r}^{2} \mathbb{V}_{i}+\left(3 r^{4}+1\right) \partial_{r} \mathbb{V}_{i}+2 r^{3} \partial_{r} \partial_{t} \mathbb{V}_{i}-r^{3} \partial_{r} \partial_{i} \mathbb{V}_{t}+r^{2}\left(\partial_{t} \mathbb{V}_{i}-\partial_{i} \mathbb{V}_{t}\right) \\
& +r\left(\partial^{2} \mathbb{V}_{i}-\partial_{i} \partial_{k} \mathbb{V}_{k}\right)-\frac{1}{2} \partial_{i} \rho+r^{2}\left(\partial_{t} \mathcal{V}_{i}-\partial_{i} \mathcal{V}_{t}\right)+r\left(\partial^{2} \mathcal{V}_{i}-\partial_{i} \partial_{k} \mathcal{V}_{k}\right) \\
& +12 \kappa r^{2} \epsilon^{i j k}\left(\frac{1}{r^{3}} \rho_{5} \partial_{j} \mathcal{V}_{k}+\frac{1}{r^{3}} \rho_{5} \partial_{j} \mathbb{V}_{k}+\partial_{r} \mathbb{A}_{t} \partial_{j} \mathcal{V}_{k}+\partial_{r} \mathbb{A}_{t} \partial_{j} \mathbb{V}_{k}\right) \\
& -12 \kappa r^{2} \epsilon^{i j k} \partial_{r} \mathbb{A}_{j}\left[\left(\partial_{t} \mathcal{V}_{k}-\partial_{k} \mathcal{V}_{t}\right)+\left(\partial_{t} \mathbb{V}_{k}-\partial_{k} \mathbb{V}_{t}\right)+\frac{1}{2 r^{2}} \partial_{k} \rho\right] \\
& -12 \kappa r^{2} \epsilon^{i j k}\left\{\partial_{r} \mathbb{V}_{j}\left[\left(\partial_{t} \mathbb{A}_{k}-\partial_{k} \mathbb{A}_{t}\right)+\frac{1}{2 r^{2}} \partial_{k} \rho_{5}\right]-\partial_{j} \mathbb{A}_{k}\left(\partial_{r} \mathbb{V}_{t}+\frac{1}{r^{3}} \rho\right)\right\} \\
0= & r^{3} \partial_{r}^{2} \mathbb{A}_{t}+3 r^{2} \partial_{r} \mathbb{A}_{t}+r \partial_{r} \partial_{k} \mathbb{A}_{k}+12 \kappa \epsilon^{i j k}\left[\partial_{r} \mathbb{V}_{i}\left(\partial_{j} \mathcal{V}_{k}+\partial_{j} \mathbb{V}_{k}\right)+\partial_{r} \mathbb{A}_{i} \partial_{j} \mathbb{A}_{k}\right], \\
0= & \left(r^{5}-r\right) \partial_{r}^{2} \mathbb{A}_{i}+\left(3 r^{4}+1\right) \partial_{r} \mathbb{A}_{i}+2 r^{3} \partial_{r} \partial_{t} \mathbb{A}_{i}-r^{3} \partial_{r} \partial_{i} \mathbb{A}_{t}+r^{2}\left(\partial_{t} \mathbb{A}_{i}-\partial_{i} \mathbb{A}_{t}\right) \\
& +r\left(\partial^{2} \mathbb{A}_{i}-\partial_{i} \partial_{k} \mathbb{A}_{k}\right)-\frac{1}{2} \partial_{i} \rho_{5}+12 \kappa r^{2} \epsilon^{i j k}\left(\partial_{j} \mathcal{V}_{k}+\partial_{j} \mathbb{V}_{k}\right)\left(\partial_{r} \mathbb{V}_{t}+\frac{1}{r^{3}} \rho\right) \\
& -12 \kappa r^{2} \epsilon^{i j k} \partial_{r} \mathbb{V}_{j}\left[\left(\partial_{t} \mathcal{V}_{k}-\partial_{k} \mathcal{V}_{t}\right)+\left(\partial_{t} \mathbb{V}_{k}-\partial_{k} \mathbb{V}_{t}\right)+\frac{1}{2 r^{2}} \partial_{k} \rho\right] \\
& -12 \kappa r^{2} \epsilon^{i j k}\left\{\partial_{r} \mathbb{A}_{j}\left[\left(\partial_{t} \mathbb{A}_{k}-\partial_{k} \mathbb{A}_{t}\right)+\frac{1}{2 r^{2}} \partial_{k} \rho_{5}\right]-\partial_{j} \mathbb{A}_{k}\left(\partial_{r} \mathbb{A}_{t}+\frac{1}{r^{3}} \rho_{5}\right)\right\}
\end{aligned}
$$

In the next section we will present solutions to (3.24)-(3.27) under approximation discussed in the Introduction.

\section{Nonlinear chiral transport}

In this section, we initially explore generic structure of the vector and axial currents (1.2) as emerges within the holographic model of section 3. No assumptions will be made regarding the charge densities $\rho, \rho_{5}$ and external fields $\vec{E}, \vec{B}$. While we are not able to solve the dynamical equations (3.24)-(3.27) analytically, we can advance by rewriting them in integral 
forms and extract near-boundary asymptotic expansion for the corrections $\mathbb{V}_{\mu}$ and $\mathbb{A}_{\mu}$. The procedure is rather tedious. Hence all the details are moved to appendix A. Via (3.16), the near-boundary asymptotic behaviors (A.1)-(A.4) yield the results (2.11), (2.12) with $\delta \vec{J}$ and $\delta \vec{J}_{5}$ formally given by (A.7). As is clear from (A.5), (A.6), $\delta \vec{J}$ and $\delta \vec{J}_{5}$ are composed of higher derivative terms involving $\vec{E}, \vec{B}$ and $\rho, \rho_{5}$.

Now we continue with the gradient expansion of $\delta \vec{J}$ and $\delta \vec{J}_{5}$. Within the hydrodynamic limit, the dynamical equations (3.24)-(3.27) are solved perturbatively. Let us introduce a formal expansion parameter $\lambda$ by $\partial_{\mu} \rightarrow \lambda \partial_{\mu}$, which counts order of the gradient expansion. Then, $\mathbb{V}_{\mu}$ and $\mathbb{A}_{\mu}$ could be expanded in powers of $\lambda$,

$$
\mathbb{V}_{\mu}=\sum_{n=1}^{\infty} \lambda^{n} \mathbb{V}_{\mu}^{[n]}, \quad \quad \mathbb{A}_{\mu}=\sum_{n=1}^{\infty} \lambda^{n} \mathbb{A}_{\mu}^{[n]} .
$$

We remind the reader that for this study, $\vec{E}$ and $\vec{B}$ are considered to be of $\mathcal{O}\left(\lambda^{1}\right)$. At each order in $\lambda, \mathbb{V}_{\mu}^{[n]}$ and $\mathbb{A}_{\mu}^{[n]}$ obey a system of ODEs, which could be analytically solved via direct integration over $r$. We list the results for $\mathbb{V}_{\mu}^{[n]}$ and $\mathbb{A}_{\mu}^{[n]}$ up to $n=2$ in (A.8)-(A.16).

Inserting the first order results (A.8)-(A.10) into (A.5)-(A.7) produces the second order results for $\delta \vec{J}$ and $\delta \vec{J}_{5}$, as summarised in (2.15), (2.16). The results (A.8), (A.13), (A.14) also lead to the expressions for the chemical potentials, as summarised in (2.21), (2.22).

With the second order corrections $\mathbb{V}_{\mu}^{[2]}$ and $\mathbb{A}_{\mu}^{[2]}$ (A.13)-(A.16), we obtain the third order results $\delta \vec{J}^{[3]}$ and $\delta \vec{J}_{5}^{[3]}$. However, nonlinearity makes such calculations rather involved and the number of various terms is very large. For the sake of presentation, we have split the third order corrections into terms that are linear in either $\rho$ or $\rho_{5}$, and the rest.

The linear in the charge densities parts of $\delta \vec{J}^{[3]}$ and $\delta \vec{J}_{5}^{[3]}$, denoted as $\delta \vec{J}^{[3] l}$ and $\delta \vec{J}_{5}^{[3] l}$, are already presented through (2.31), (2.32). These terms are the ones that contribute to the gapless waves propagating in the chiral medium. We focus on the case with constant external fields only. Consider a plane wave ansatz for the charge densities

$$
\delta \rho=e^{-i(\omega t-\vec{q} \cdot \vec{x})} \delta \tilde{\rho}, \quad \delta \rho_{5}=e^{-i(\omega t-\vec{q} \cdot \vec{x})} \delta \tilde{\rho}_{5} .
$$

Then, the continuity equations (1.1) with the constitutive relations (2.11), (2.12), (2.15), (2.16), (2.31), (2.32) turn into

$$
a \delta \tilde{\rho}+b \delta \tilde{\rho}_{5}=0, \quad b \delta \tilde{\rho}+a \delta \tilde{\rho}_{5}=12 \kappa(\overrightarrow{\mathbf{E}} \cdot \overrightarrow{\mathbf{B}}) .
$$

The explicit expressions for $a$ and $b$ are

$$
\begin{aligned}
a= & -i \omega+\frac{1}{2} q^{2}+18(1-2 \log 2) \kappa^{2} q^{2} \mathbf{B}^{2}-\frac{3 \pi^{2}}{4} \kappa^{2} q^{2} \mathbf{E}^{2}+9(\pi-2 \log 2) \kappa^{2}(\vec{q} \cdot \overrightarrow{\mathbf{B}})^{2} \\
& +\frac{3 \pi^{2}}{4} \kappa^{2}(\vec{q} \cdot \overrightarrow{\mathbf{E}})^{2}+i \frac{\pi}{8} \omega q^{2}-\frac{\pi^{2}}{48} \omega^{2} q^{2}-\frac{1}{16}(\pi-2 \log 2) q^{4}+i 36 \log 2 \kappa^{2}(\vec{q} \cdot \overrightarrow{\mathbf{S}}) \\
& -\left(18 \mathcal{C}+\frac{21 \pi^{2}}{8}\right) \kappa^{2} \omega(\vec{q} \cdot \overrightarrow{\mathbf{S}}), \\
b= & i 6 \kappa(\vec{q} \cdot \overrightarrow{\mathbf{B}})-i \frac{3}{4}(\pi-2 \log 2) \kappa q^{2}(\vec{q} \cdot \overrightarrow{\mathbf{B}})+i 216(1-2 \log 2) \kappa^{3} \mathbf{B}^{2}(\vec{q} \cdot \overrightarrow{\mathbf{B}}) \\
& -\frac{3}{2}(\pi+2 \log 2) \kappa \omega(\vec{q} \cdot \overrightarrow{\mathbf{B}})-i \frac{1}{8}\left(24 \mathcal{C}+\pi^{2}+6 \log ^{2} 2\right) \kappa \omega^{2}(\vec{q} \cdot \overrightarrow{\mathbf{B}}) \\
& -i \frac{3}{4}(\pi-2 \log 2) \kappa q^{2}(\vec{q} \cdot \overrightarrow{\mathbf{B}})+i 9 \pi^{2} \kappa^{3}\left[(\overrightarrow{\mathbf{B}} \cdot \overrightarrow{\mathbf{E}})(\vec{q} \cdot \overrightarrow{\mathbf{E}})-\mathbf{E}^{2}(\vec{q} \cdot \overrightarrow{\mathbf{B}})\right],
\end{aligned}
$$


where the Poynting vector $\overrightarrow{\mathbf{S}}=\overrightarrow{\mathbf{E}} \times \overrightarrow{\mathbf{B}}$. For $\omega, q \ll 1$, the dispersion equation (4.3) can be solved perturbatively, leading to the $\mathbf{B} / \mathbf{E}$-corrected dispersion relation (2.35).

Finally, we turn to terms that are nonlinear in the charge densities in the third order results $\delta \vec{J}^{[3]}$ and $\delta \vec{J}_{5}^{[3]}$. We denote them as $\delta \vec{J}^{[3] n l}$ and $\delta \vec{J}_{5}^{[3] n l}$ :

$$
\begin{aligned}
\delta \vec{J}^{[3] n l}= & \tau_{23}\left(\rho^{2}+\rho_{5}^{2}\right) \partial_{t} \vec{\nabla} \times \vec{B}+\tau_{24} \rho_{5}\left(\rho_{5}^{2}+3 \rho^{2}\right) \vec{\nabla}^{2} \vec{B}+\tau_{25} \partial_{t} \vec{H}+\tau_{26}\left(\partial_{t} \rho_{5} \vec{\nabla} \rho_{5}+\partial_{t} \rho \vec{\nabla} \rho\right) \times \vec{B} \\
& +\tau_{27}\left(\rho_{5} \partial_{t} \vec{\nabla} \rho_{5}+\rho \partial_{t} \vec{\nabla} \rho\right) \times \vec{B}+\tau_{28}\left(\rho_{5} \partial_{t} \rho_{5}+\rho \partial_{t} \rho\right) \vec{\nabla} \times \vec{B}+\tau_{29}\left(\rho_{5} \vec{\nabla} \rho_{5}+\rho \vec{\nabla} \rho\right) \times \partial_{t} \vec{B} \\
& +\tau_{30} 24 \kappa \rho \rho_{5} \vec{\nabla} \times \vec{S}+\tau_{31}\left(\rho_{5} \vec{\nabla} \rho+\rho \vec{\nabla} \rho_{5}\right) \times \vec{S}+\tau_{32}\left(\vec{\nabla} \rho_{5} \times \partial_{t} \vec{\nabla} \rho+\vec{\nabla} \rho \times \partial_{t} \vec{\nabla} \rho_{5}\right) \\
& +\tau_{33} 2 \rho \rho_{5} \vec{B} \times \partial_{t} \vec{B}+\tau_{34} 2 \rho \rho_{5} \vec{E} \times(\vec{\nabla} \times \vec{B})+\tau_{35}\left[\rho_{5} \vec{\nabla} \times\left(\vec{E} \times \vec{\nabla} \rho_{5}\right)+\left(\rho_{5} \rightarrow \rho\right)\right] \\
& +\tau_{36}\left[\vec{\nabla} \rho \times(\vec{E} \times \vec{\nabla} \rho)+\left(\rho \rightarrow \rho_{5}\right)\right]+\tau_{37}\left[2 \rho \rho_{5} \vec{\nabla} \rho+\left(\rho^{2}+\rho_{5}^{2}\right) \vec{\nabla} \rho_{5}\right] \times(\vec{\nabla} \times \vec{B}) \\
& +\tau_{38}\left(\rho_{5} \vec{\nabla} \times \vec{H}+\rho \vec{\nabla} \times \vec{H}_{a}\right)+\tau_{39}\left(\vec{\nabla} \rho_{5} \times \vec{H}+\vec{\nabla} \rho \times \vec{H}_{a}\right)+\tau_{40} \vec{E} \times \vec{H}_{a}, \\
\delta \vec{J}_{5}^{[3] n l}= & \tau_{23} 2 \rho \rho_{5} \partial_{t} \vec{\nabla} \times \vec{B}+\tau_{24} \rho\left(\rho^{2}+3 \rho_{5}^{2}\right) \vec{\nabla}^{2} \vec{B}+\tau_{25} \partial_{t} \vec{H}_{a}+\tau_{26}\left(\partial_{t} \rho \vec{\nabla} \rho_{5}+\partial_{t} \rho_{5} \vec{\nabla} \rho\right) \times \vec{B} \\
& +\tau_{27}\left(\rho \partial_{t} \vec{\nabla} \rho_{5}+\rho_{5} \partial_{t} \vec{\nabla} \rho\right) \times \vec{B}+\tau_{28}\left(\rho \partial_{t} \rho_{5}+\rho_{5} \partial_{t} \rho\right) \vec{\nabla} \times \vec{B}+\tau_{29}\left(\rho \vec{\nabla} \rho_{5}+\rho_{5} \vec{\nabla} \rho\right) \times \partial_{t} \vec{B} \\
& +\tau_{30} 12 \kappa\left(\rho^{2}+\rho_{5}^{2}\right) \vec{\nabla} \times \vec{S}+\tau_{31}\left(\rho \vec{\nabla} \rho+\rho_{5} \vec{\nabla} \rho_{5}\right) \times \vec{S}+\tau_{32}\left(\vec{\nabla} \rho \times \partial_{t} \vec{\nabla} \rho+\vec{\nabla} \rho_{5} \times \partial_{t} \vec{\nabla} \rho_{5}\right) \\
& +\tau_{33}\left(\rho^{2}+\rho_{5}^{2}\right) \vec{B} \times \partial_{t} \vec{B}+\tau_{34}\left(\rho^{2}+\rho_{5}^{2}\right) \vec{E} \times(\vec{\nabla} \times \vec{B})+\tau_{35}\left[\rho \vec{\nabla} \times\left(\vec{E} \times \vec{\nabla} \rho_{5}\right)+\left(\rho_{5} \leftrightarrow \rho\right)\right] \\
& +\tau_{36}\left[\vec{\nabla} \rho_{5} \times(\vec{E} \times \vec{\nabla} \rho)+\left(\rho \leftrightarrow \rho_{5}\right)\right]+\tau_{37}\left[2 \rho \rho_{5} \vec{\nabla} \rho_{5}+\left(\rho^{2}+\rho_{5}^{2}\right) \vec{\nabla} \rho\right] \times(\vec{\nabla} \times \vec{B}) \\
& +\tau_{38}\left(\rho \vec{\nabla} \times \vec{H}+\rho_{5} \vec{\nabla} \times \vec{H}_{a}\right)+\tau_{39}\left(\vec{\nabla} \rho \times \vec{H}+\vec{\nabla} \rho_{5} \times \overrightarrow{H_{a}}\right)+\tau_{40} \vec{E} \times \vec{H},
\end{aligned}
$$

where

$$
\vec{S}=\vec{E} \times \vec{B}, \quad \vec{H}=\vec{B} \times\left(\rho_{5} \vec{\nabla} \rho_{5}+\rho \vec{\nabla} \rho\right), \quad \vec{H}_{a}=\vec{B} \times\left(\rho \vec{\nabla} \rho_{5}+\rho_{5} \vec{\nabla} \rho\right) .
$$

All $\tau_{i}$ 's in (4.6), (4.7) are computed analytically and the results are deposited in appendix A, see (A.51)-(A.68). Below we give simple explanation for each term in (4.6), (4.7).

The TC $\tau_{23}$ corresponds to anomalous corrections to the relaxation term in the magnetic conductivity $\sigma_{m}$ of $[24,111]$. The analytical result for $\tau_{23}$ was unknown in [24]. The $\tau_{28}$-term is a nonlinear correction to the magnetic current ( $\sigma_{m}$-term of [111]), and relies on time-varying densities. $\tau_{37}$ corresponds to a mixing effect between the charge diffusion and magnetic current. The TC $\tau_{30}$ is due to spatial inhomogeneity of e/m energy flux and is an analog of the magnetic conductivity.

The $\tau_{24}$-term stands for second order expansion of the CME conductivity $\sigma_{\chi}$ of [24] and was first computed there. $\tau_{25}$ is the relaxation term for the second order Hall diffusion current (see the $\mathcal{D}_{H}^{0}$-term in $\left.(2.23),(2.24)\right)$. The $\tau_{26}, \tau_{27}, \tau_{29}$-terms rely on the time inhomogeneity of charge densities or magnetic field and could be thought of as extension of the Hall diffusion current. The TC $\tau_{31}$ is related to the e/m energy flux and also generalises the Hall diffusion current.

$\tau_{32}$ is composed of spatial gradient of charge densities and corresponds to nonlinear charge diffusion process. The $\tau_{36}, \tau_{39}$-terms are $\mathrm{e} / \mathrm{m}$ field corrections to the nonlinear charge diffusions. The last TC $\tau_{40}$ is a nonlinear in $E, B$ correction to the charge diffusions. The terms $\tau_{33}, \tau_{34}$ are nonlinear in densities corrections to $\tau_{21}, \tau_{22}$. 
$\tau_{35}$ is the third order extension of the anomalous chiral Hall effect, i.e., $\sigma_{a \chi}^{0} H_{\text {-term }}$ in $(2.23),(2.24)$. In [96], we will perform a systematic resummation for certain transports and the term $\tau_{35}$ will be generalised into a TCF. Similarly, $\tau_{38}$ can be simply taken as the magnetic analogue of $\tau_{35}$ and will be extended to a TCF in [96].

Finally, let us mention the dissipative nature for each term in the third order results (4.6), (4.7). Via the criterion of $\mathcal{T}$-symmetry, the TCs $\tau_{24}, \tau_{30-34}, \tau_{37-40}$ are $\mathcal{T}$-even and are thus non-dissipative. The remaining terms are all dissipative.

\section{Conclusion}

In this work, we have continued exploration of nonlinear chiral anomaly-induced transport phenomena based on a holographic model with two U(1) fields interacting via a ChernSimons term. For a finite temperature system, we constructed off-shell constitutive relations for the vector/axial currents. A detailed report on our new results could be found in the Summary section. Here they are in brief:

- We demonstrated that both CME and CSE get corrected by higher derivative terms, see (2.11), (2.12). In the hydrodynamic limit, we analytically calculated those gradient corrections up to third order. Comparison with the CKT was presented. New third order results, particularly (2.31), (2.32), extend those that were initially considered in $[24,25]$ and reveal novel effects associated with time-dependence or inhomogeneities of the charge densities and external fields.

- Among new results worth highlighting, in weak field approximation the charge diffusion constant $\mathcal{D}_{0}$ was found to receive negative anomaly-induced $\mathbf{E}^{2}$ - and $\mathbf{B}^{2}$ corrections (2.34). It is very interesting to explore the dependence of $\mathcal{D}_{0}$ on the $\mathrm{e} / \mathrm{m}$ fields beyond the weak field approximation, that is non-perturbatively. Of particular interest would be a strong field limit. We are pursuing this line of study in the forthcoming paper [117] (see also [95] for similar study but in a different holographic model $\left.^{9}\right)$.

- Another result we found to be of interest is that the chiral medium is shown to support three types of collective modes: CMW (propagating along $\overrightarrow{\mathbf{B}}$ ), CEW propagating along $\overrightarrow{\mathbf{E}}$, and a new one, chiral Hall density wave, propagating orthogonal to the other two, that is, along the energy flux $\overrightarrow{\mathbf{E}} \times \overrightarrow{\mathbf{B}}$.

The follow up paper [96] focuses on another set of approximations. Instead of considering a fixed order gradient expansion adopted here, we compute some TCFs in nonlinear chiral transport phenomena. More specifically, the external electromagnetic fields are assumed to be constant and weak, while the charge densities are split into constant backgrounds and small inhomogeneous fluctuations. The setup is similar to that of [24], but in [96] as opposed to [24], gradient resummation is performed for terms that are linear both in the charge density fluctuations and external fields.

\footnotetext{
${ }^{9}$ In [95] the effect of non-perturbative magnetic field on the speed of CMW and diffusion constant was induced by nonlinear DBI action, which is quite different from the model in [117].
} 
We have found a wealth of non-linear phenomena all induced entirely by the chiral anomaly. An important next step in deriving a full chiral MHD would be to abandon the probe limit adopted in this paper and include the dynamics of a neutral flow as well. This will bring into the picture additional effects such as thermoelectric conductivities, normal Hall current, the chiral vortical effect [119, 120], and some nonlinear effects discussed in [103]. We plan to address these in the future.

\section{A Supplement for section 4}

In this appendix, we collect all calculational details omitted in section 4. Regarding the general structure of the constitutive relations of the vector/axial currents, we present the integral versions of the bulk dynamical equations and explore near boundary asymptotics. We further derive the gradient expansion, and compute analytically all TCs, up to third order.

The dynamical equations (3.24)-(3.27) can be directly integrated over $r$, resulting in the following integral forms

$$
\begin{aligned}
\mathbb{V}_{t}= & -\int_{r}^{\infty} \frac{d x}{x^{3}} \int_{x}^{\infty} d y\left\{y \partial_{y} \partial_{k}\left(\mathbb{V}_{k}+\frac{E_{k}}{y}\right)+12 \kappa \epsilon^{i j k} \partial_{y} \mathbb{A}_{i}\left(\partial_{j} \mathcal{V}_{k}+\partial_{j} \mathbb{V}_{k}\right)\right. \\
& \left.+12 \kappa \epsilon^{i j k} \partial_{y} \mathbb{V}_{i} \partial_{j} \mathbb{A}_{k}\right\}+\partial_{k} E_{k}\left(\frac{\log r}{2 r^{2}}+\frac{1}{4 r^{2}}\right) \\
& \stackrel{r \rightarrow \infty}{\longrightarrow} \partial_{k} E_{k}\left(\frac{\log r}{2 r^{2}}+\frac{1}{4 r^{2}}\right)+\mathcal{O}\left(\frac{\log r}{r^{3}}\right), \\
\mathbb{V}_{i}= & -\int_{r}^{\infty} \frac{x d x}{x^{4}-1}\left\{-\partial_{t} E_{i} \log x+\frac{x-1}{2 x} \partial_{i} \rho+(x-1) E_{i}+\epsilon^{i j k} \partial_{j} B_{k} \log x-12 \kappa B_{i}\right. \\
& \stackrel{r \rightarrow \infty}{\longrightarrow}\left(\frac{\log r}{2 r^{2}}+\frac{1}{4 r^{2}}\right)\left(\partial_{t} E_{i}-\partial_{k} \mathcal{F}_{i k}\right)-\frac{1}{4 r^{2}} \partial_{i} \rho+\left(-\frac{1}{r}+\frac{1}{2 r^{2}}\right) E_{i}+\frac{6 \kappa \mu_{5} B_{i}}{r^{2}} \\
& -\stackrel{G_{i}(x=\infty)}{2 r^{2}}+\mathcal{O}\left(\frac{\log r}{r^{3}}\right), \\
\mathbb{A}_{t}= & -\int_{r}^{\infty} \frac{d x}{x^{3}} \int_{x}^{\infty}\left\{y \partial_{y} \partial_{k} \mathbb{A}_{k}(y)+12 \kappa \epsilon^{i j k} \partial_{y} \mathbb{V}_{i}\left(\partial_{j} \mathcal{V}_{k}+\partial_{j} \mathbb{V}_{k}\right)+12 \kappa \epsilon^{i j k} \partial_{y} \mathbb{A}_{i} \partial_{j} \mathbb{A}_{k}\right\} \\
& \stackrel{r \rightarrow \infty}{\longrightarrow} \mathcal{O}\left(\frac{1}{r^{3}}\right), \\
\mathbb{A}_{i}= & -\int_{r}^{\infty} \frac{x d x}{x^{4}-1}\left\{\frac{x-1}{2 x} \partial_{i} \rho_{5}-12 \kappa B_{i}\left[\mu+\mathbb{V}_{t}(x)-\frac{\rho}{2 x^{2}}\right]+H_{i}(x)\right\} \\
& \stackrel{r \rightarrow \infty}{\longrightarrow}-\frac{1}{4 r^{2}} \partial_{i} \rho_{5}+\frac{6 \kappa B_{i} \mu}{r^{2}}-\frac{1}{2 r^{2}} H_{i}(x=\infty)+\mathcal{O}\left(\frac{1}{r^{3}}\right),
\end{aligned}
$$

where $\mu$ and $\mu_{5}$ are the chemical potentials defined in (3.23). We have also provided 
asymptotic expansions near the boundary $r=\infty$. The functions $G_{i}(x)$ and $H_{i}(x)$ are

$$
\begin{aligned}
G_{i}(x)=-\int_{1}^{x} d y\{ & 2 y \partial_{y} \partial_{t}\left[\mathbb{V}_{i}(y)+\frac{E_{i}}{y}\right]+\partial_{t}\left(\mathbb{V}_{i}(y)+\frac{E_{i}}{y}\right)-y \partial_{y} \partial_{i} \mathbb{V}_{t}-\partial_{i} \mathbb{V}_{t} \\
& +\frac{1}{y}\left(\partial^{2} \mathbb{V}_{i}-\partial_{i} \partial_{k} \mathbb{V}_{k}\right)+12 \kappa \epsilon^{i j k}\left[\frac{1}{y^{3}} \rho_{5} \partial_{j} \mathbb{V}_{k}+\partial_{y} \mathbb{A}_{t} \partial_{j} \mathbb{V}_{k}\right] \\
& -12 \kappa \epsilon^{i j k} \partial_{y} \mathbb{A}_{j}\left[\left(\partial_{t} \mathbb{V}_{k}-\partial_{k} \mathbb{V}_{t}\right)+\frac{1}{2 y^{2}} \partial_{k} \rho-E_{k}\right] \\
& -12 \kappa \epsilon^{i j k} \partial_{y} \mathbb{V}_{j}\left[\left(\partial_{t} \mathbb{A}_{k}-\partial_{k} \mathbb{A}_{t}\right)+\frac{1}{2 y^{2}} \partial_{k} \rho_{5}\right] \\
& \left.+12 \kappa \epsilon^{i j k}\left(\frac{1}{y^{3}} \rho \partial_{j} \mathbb{A}_{k}+\partial_{y} \mathbb{V}_{t} \partial_{j} \mathbb{A}_{k}\right)\right\}, \\
H_{i}(x)=-\int_{1}^{x} d y\{ & 2 y \partial_{y} \partial_{t} \mathbb{A}_{i}-y \partial_{y} \partial_{i} \mathbb{A}_{t}+\left(\partial_{t} \mathbb{A}_{i}-\partial_{i} \mathbb{A}_{t}\right)+\frac{1}{y}\left(\partial^{2} \mathbb{A}_{i}-\partial_{i} \partial_{k} \mathbb{A}_{k}\right)+12 \kappa \epsilon^{i j k} \\
& \times\left[\frac{\rho}{y^{3}} \partial_{j} \mathbb{V}_{k}+\partial_{y} \mathbb{V}_{t} \partial_{j} \mathbb{V}_{k}\right]-12 \kappa \epsilon^{i j k} \partial_{y} \mathbb{V}_{j}\left[\left(\partial_{t} \mathbb{V}_{k}-\partial_{k} \mathbb{V}_{t}\right)+\frac{\partial_{k} \rho}{2 y^{2}}-E_{k}\right] \\
& -12 \kappa \epsilon^{i j k} \partial_{y} \mathbb{A}_{j}\left[\left(\partial_{t} \mathbb{A}_{k}-\partial_{k} \mathbb{A}_{t}\right)+\frac{1}{2 y^{2}} \partial_{k} \rho_{5}\right]+12 \kappa \epsilon^{i j k}\left(\frac{1}{y^{3}} \rho_{5} \partial_{j} \mathbb{A}_{k}\right. \\
& \left.\left.+\partial_{y} \mathbb{A}_{t} \partial_{j} \mathbb{A}_{k}\right)\right\} .
\end{aligned}
$$

In deriving (A.1)-(A.4), all three types of the boundary conditions, as summarized in section 3, were used to fix the integration constants. The formal solutions (A.1)-(A.4) give rise to the general results $(2.11),(2.12)$ with $\delta J^{i}$ and $\delta J_{5}^{i}$ given as

$$
\delta J^{i}=\partial_{t} E_{i}-G_{i}(x=\infty), \quad \delta J_{5}^{i}=-H_{i}(x=\infty)
$$

For generic profiles of $\rho, \rho_{5}, \vec{E}, \vec{B}$, we are not able to compute $G_{i}(x=\infty)$ and $H_{i}(x=$ $\infty)$ analytically. So, we employ the standard hydrodynamic limit and evaluate them up to third order in the gradient expansion (4.1). Perturbative solutions for $\mathbb{V}_{\mu}$ and $\mathbb{A}_{\mu}$ are collected below. At first order, $n=1$,

$$
\begin{aligned}
\mathbb{V}_{t}^{[1]} & =\mathbb{A}_{t}^{[1]}=0, \\
\mathbb{V}_{i}^{[1]} & =f_{1}(r) \partial_{i} \rho+f_{3}(r) E_{i}+f_{2}(r) \rho_{5} B_{i}, \\
\mathbb{A}_{i}^{[1]} & =f_{1}(r) \partial_{i} \rho_{5}+f_{2}(r) \rho B_{i},
\end{aligned}
$$

where

$$
\begin{aligned}
f_{1}(r) & =\frac{1}{8}\left[\log \frac{(1+r)^{2}}{1+r^{2}}+2 \arctan (r)-\pi\right], \quad f_{2}(r)=3 \kappa \log \frac{1+r^{2}}{r^{2}} \\
f_{3}(r) & =\frac{1}{4}\left[\log \frac{1+r^{2}}{(1+r)^{2}}+2 \arctan (r)-\pi\right] .
\end{aligned}
$$


At second order, $n=2$,

$$
\begin{aligned}
\mathbb{V}_{t}^{[2]}= & a_{0} \partial_{k} E_{k}+a_{1}\left(-\frac{1}{2} \partial^{2} \rho+6 \kappa B_{k} \partial_{k} \rho_{5}\right)+a_{2} 72 \kappa^{2} B^{2} \rho \\
\mathbb{A}_{t}^{[2]}= & a_{1}\left(-\frac{1}{2} \partial^{2} \rho_{5}+6 \kappa B_{k} \partial_{k} \rho-12 \kappa \vec{E} \cdot \vec{B}\right)+a_{2} 72 \kappa^{2} B^{2} \rho_{5} \\
\mathbb{V}_{i}^{[2]}= & b_{0} \epsilon^{i j k} \partial_{j} B_{k}+b_{1} \partial_{t} \partial_{i} \rho+b_{2} \partial_{t} E_{i}+b_{3} 6 \kappa \partial_{t}\left(\rho_{5} B_{i}\right)+b_{4} 3 \kappa \rho_{5} \epsilon^{i j k} \partial_{j} E_{k}+b_{5} 36 \kappa^{2} \epsilon^{i j k} \\
& \times\left[-\left(\rho^{2}+\rho_{5}^{2}\right) \partial_{j} B_{k}+\rho_{5} B_{j} \partial_{k} \rho_{5}+\rho B_{j} \partial_{k} \rho\right]+b_{6} 6 \kappa \epsilon^{i j k}\left[E_{j} \partial_{k} \rho_{5}+12 \kappa \rho B_{j} E_{k}\right] \\
& -b_{7} 36 \kappa^{2} \epsilon^{i j k}\left(\rho B_{j} \partial_{k} \rho+\rho_{5} B_{j} \partial_{k} \rho_{5}\right) \\
\mathbb{A}_{i}^{[2]}= & b_{1} \partial_{t} \partial_{i} \rho_{5}+b_{3} 6 \kappa \partial_{t}\left(\rho B_{i}\right)+b_{4} 3 \kappa \rho \epsilon^{i j k} \partial_{j} E_{k}+b_{5} 36 \kappa^{2} \epsilon^{i j k}\left(-2 \rho \rho_{5} \partial_{j} B_{k}+\rho B_{j} \partial_{k} \rho_{5}\right. \\
& \left.+\rho_{5} B_{j} \partial_{k} \rho\right)+b_{6} 6 \kappa \epsilon^{i j k}\left(E_{j} \partial_{k} \rho+12 \kappa \rho_{5} B_{j} E_{k}\right)-b_{7} 36 \kappa^{2} \epsilon^{i j k}\left[\rho_{5} B_{j} \partial_{k} \rho+\rho B_{j} \partial_{k} \rho_{5}\right],
\end{aligned}
$$

where

$$
\begin{aligned}
& a_{0}=\frac{1+2 \log r}{4 r^{2}}+\int_{r}^{\infty} \frac{d x}{x^{3}} \int_{x}^{\infty} d y \frac{y^{2}+y+1}{y\left(y^{2}+1\right)(y+1)}, \\
& a_{1}=\int_{r}^{\infty} \frac{d x}{x^{3}} \int_{x}^{\infty} \frac{y d y}{\left(y^{2}+1\right)(y+1)}, \\
& a_{2}=\int_{r}^{\infty} \frac{d x}{x^{3}} \int_{x}^{\infty} \frac{d y}{y\left(y^{2}+1\right)} \\
& b_{0}=-\int_{r}^{\infty} \frac{x d x}{x^{4}-1} \int_{1}^{x} \frac{d y}{y} \\
& b_{1}=-\int_{r}^{\infty} \frac{x d x}{x^{4}-1} \int_{1}^{x} d y\left\{-\frac{y}{\left(y^{2}+1\right)(y+1)}-\frac{1}{8}\left[\log \frac{(1+y)^{2}}{1+y^{2}}+2 \arctan (y)-\pi\right]\right\}, \\
& b_{2}=-\int_{r}^{\infty} \frac{x d x}{x^{4}-1} \int_{1}^{x} d y\left\{-\frac{2 y^{2}}{\left(y^{2}+1\right)(y+1)}-\frac{1}{4}\left[\log \frac{1+y^{2}}{(1+y)^{2}}+2 \arctan (y)-\pi\right]\right\}, \\
& b_{3}=-\int_{r}^{\infty} \frac{x d x}{x^{4}-1} \int_{1}^{x} d y\left\{\frac{2}{y^{2}+1}-\frac{1}{2} \log \frac{1+y^{2}}{y^{2}}\right\} \\
& b_{4}=-\int_{r}^{\infty} \frac{x d x}{x^{4}-1} \int_{1}^{x} d y\left\{-\frac{1}{y^{3}}\left[\log \frac{1+y^{2}}{(1+y)^{2}}+2 \arctan (y)-\pi\right]\right\}, \\
& b_{5}=-\int_{r}^{\infty} \frac{x d x}{x^{4}-1} \int_{1}^{x} d y \frac{1}{y^{3}} \log \frac{1+y^{2}}{y^{2}} \\
& b_{6}=-\int_{r}^{\infty} \frac{x d x}{x^{4}-1} \int_{1}^{x} \frac{d y}{y\left(y^{2}+1\right)}, \\
& b_{7}=-\int_{r}^{\infty} \frac{x d x}{x^{4}-1} \int_{1}^{x} \frac{d y}{y^{3}\left(y^{2}+1\right)} .
\end{aligned}
$$

Substituting the first order solutions (A.8), (A.9), (A.10) into (A.5), (A.6) generates the second order results $(2.15),(2.16)$. The chemical potentials $(2.21),(2.22)$ are obtained similarly by substituting the results (A.8), (A.13), (A.14) into (3.23). Finally, the solutions (A.8)-(A.16) give rise to the third order corrections (2.31), (2.32) with the transport 
coefficients $\tau_{1-40}$ as

$$
\begin{aligned}
& \tau_{1}=\int_{1}^{\infty} d y\left[2 y \partial_{y} b_{1}(y)+b_{1}(y)\right]=-\frac{\pi^{2}}{48} \\
& \tau_{2}=\int_{1}^{\infty} d y \frac{1}{2}\left[y \partial_{y} a_{1}(y)+a_{1}(y)\right]=-\frac{1}{16}(\pi-2 \log 2), \\
& \tau_{3}=\int_{1}^{\infty} d y\left[2 y \partial_{y} b_{2}(y)+b_{2}(y)\right]=-\frac{\pi^{2}}{24}, \\
& \tau_{4}=-\int_{1}^{\infty} d y\left[y \partial_{y} a_{0}(y)+a_{0}(y)\right]=\frac{1}{8}(\pi+2 \log 2), \\
& \tau_{5}=\int_{1}^{\infty} d y\left[\frac{f_{3}(y)}{y}+\left(y \partial_{y}+1\right) a_{0}(y)+\left(2 y \partial_{y}+1\right) b_{0}(y)\right]=-\frac{1}{8}\left(\pi-\frac{\pi^{2}}{2}+2 \log 2\right), \\
& \tau_{6}=\int_{1}^{\infty} d y 6 \kappa\left[2 y \partial_{y} b_{3}(y)+b_{3}(y)\right]=\frac{1}{8} \kappa\left(24 \mathcal{C}+\pi^{2}+6 \log ^{2} 2\right), \\
& \tau_{7}=\int_{1}^{\infty} d y 3 \kappa\left(2 y \partial_{y}+1\right)\left[4 b_{3}(y)+b_{4}(y)\right]=9 \kappa \mathcal{C}+\frac{5}{16} \kappa \pi^{2}+\frac{3}{2} \kappa \log ^{2} 2, \\
& \tau_{8}=\int_{1}^{\infty} d y\left\{3 \kappa\left(2 y \partial_{y}+1\right)\left[2 b_{3}(y)+b_{4}(y)\right]+12 \kappa \frac{b_{2}(y)}{y^{3}}\right\}=\kappa\left(6 \mathcal{C}+\frac{1}{4} \pi^{2}\right), \\
& \tau_{9}=\int_{1}^{\infty} d y \frac{f_{2}(y)}{y}=\frac{1}{8} \kappa \pi^{2}, \\
& \tau_{10}=\int_{1}^{\infty}\left[\frac{f_{2}(y)}{y}-12 \kappa \frac{b_{0}(y)}{y^{3}}\right]=\frac{1}{4} \kappa \pi^{2}, \\
& \tau_{11}=\int_{1}^{\infty}\left[\frac{2 f_{2}(y)}{y}+12 \kappa \frac{\partial_{y} b_{0}(y)}{2 y^{2}}\right]=\frac{5}{16} \kappa \pi^{2}, \\
& \tau_{12}=-\int_{1}^{\infty}\left[6 \kappa\left[y \partial_{y} a_{1}(y)+a_{1}(y)\right]+\frac{f_{2}(y)}{y}\right]=\frac{1}{8} \kappa\left(6 \pi-12 \log 2-\pi^{2}\right), \\
& \tau_{13}=-\int_{1}^{\infty} 72 \kappa^{2}\left[y \partial_{y} a_{2}(y)+a_{2}(y)\right]=18 \kappa^{2}(2 \log 2-1), \\
& \tau_{14}=-\int_{1}^{\infty} d y 72 \kappa^{2} \partial_{y} b_{6}(y)=-\frac{3}{4} \kappa^{2} \pi^{2} \\
& \tau_{15}=\int_{1}^{\infty}\left\{6 \kappa\left[2 y \partial_{y} b_{6}(y)+b_{6}(y)\right]-12 \kappa\left[f_{1}(y) \partial_{y} f_{3}(y)+\partial_{y} b_{1}(y)\right]\right\} \\
& =\frac{3}{2} \kappa \mathcal{C}+\frac{5}{32} \kappa \pi^{2}, \\
& \tau_{16}=\int_{1}^{\infty} d y 12 \kappa\left[\partial_{y} b_{1}(y)+\partial_{y}\left(f_{1}(y) f_{3}(y)\right)-\frac{\partial_{y} b_{2}(y)}{2 y^{2}}\right] \\
& =-\frac{1}{8} \kappa\left[12 \mathcal{C}+\frac{\pi^{2}}{4}-6 \log ^{2} 2\right], \\
& \tau_{17}=-\int_{1}^{\infty} d y 36 \kappa^{2} \partial_{y} b_{4}(y)=-\frac{3}{8} \kappa^{2}\left(48 \mathcal{C}-\pi^{2}\right), \\
& \tau_{18}=\int_{1}^{\infty} d y\left[72 \kappa^{2}\left(2 y \partial_{y} b_{6}(y)+b_{6}(y)\right)-12 \kappa f_{3}(y) \partial_{y} f_{2}(y)\right]=\frac{3 \pi^{2}}{2} \kappa^{2}, \\
& \tau_{19}=\int_{1}^{\infty} d y 864 \kappa^{3} \partial_{y} b_{6}(y)=9 \kappa^{3} \pi^{2},
\end{aligned}
$$




$$
\begin{aligned}
& \tau_{20}=-\int_{1}^{\infty} d y 12 \kappa\left[6 \kappa \partial_{y} b_{3}(y)+\partial_{y}\left(f_{2}(y) f_{3}(y)\right)\right]=-\frac{3}{8} \kappa^{2}\left(48 \mathcal{C}+3 \pi^{2}\right), \\
& \tau_{21}=\int_{1}^{\infty} d y 12 \kappa \partial_{y} b_{0}(y)=\frac{3}{8} \kappa \pi^{2}, \\
& \tau_{22}=-2 \tau_{14}=-\frac{3}{2} \kappa(\pi-2 \log 2), \\
& \tau_{23}=\int_{1}^{\infty} d y\left\{\frac{36 \kappa^{2}}{y^{3}}\left[2 b_{3}(y)+b_{4}(y)\right]-36 \kappa^{2}\left[2 \partial_{y} b_{5}(y)+b_{5}(y)\right]\right\}=\frac{3}{2} \kappa^{2}\left(\pi^{2}-12 \log 2\right), \\
& \tau_{24}=\int_{1}^{\infty} d y 432 \kappa^{3}\left[\frac{1}{y^{3}} b_{5}(y)\right]=-108 \kappa^{3}(\log 2-1)^{2}, \\
& \tau_{25}=\int_{1}^{\infty} d y\left\{36 \kappa^{2}\left(2 \partial_{y}+1\right)\left[b_{5}(y)-b_{7}(y)\right]+12 \kappa\left[f_{2}(y) \partial_{y} f_{1}(y)-f_{1}(y) \partial_{y} f_{2}(y)\right]\right. \\
& \left.-\frac{18 \kappa^{2}}{y^{3}}\left[y \partial_{y} b_{4}(y)+2 y \partial_{y} b_{3}(y)+4 b_{3}(y)+2 b_{4}(y)\right]\right\} \\
& =\frac{3}{16} \kappa^{2}\left[-144 \mathcal{C}+13 \pi^{2}+72 \log ^{2} 2+12 \pi(9 \log 2-4)\right] \text {, } \\
& \tau_{26}=-\int_{1}^{\infty} d y\left\{\frac{18 \kappa^{2}}{y^{3}}\left[4 b_{3}(y)+2 b_{4}(y)+y \partial_{y} b_{4}(y)\right]+12 \kappa f_{1}(y) \partial_{y} f_{2}(y)\right\} \\
& =\frac{9}{4} \kappa^{2}[-8 \mathcal{C}+(8+5 \pi) \log 2], \\
& \tau_{27}=-\int_{1}^{\infty} d y\left\{\frac{18 \kappa^{2}}{y^{3}}\left[2 b_{4}(y)+2 y \partial_{y} b_{3}(y)+y \partial_{y} b_{4}(y)\right]-12 \kappa f_{2}(y) \partial_{y} f_{1}(y)\right\} \\
& =\frac{9}{16} \kappa^{2}\left[48 \mathcal{C}+\pi^{2}-4(8+7 \pi) \log 2\right], \\
& \tau_{28}=\int_{1}^{\infty} d y 72 \kappa^{2}\left[\frac{b_{3}(y)}{y^{3}}-\left[2 \partial_{y}+1\right] b_{5}(y)\right]=\frac{3}{4} \kappa^{2}[\pi(5 \pi-12)+12(\pi-2) \log 2], \\
& \tau_{29}=-\int_{1}^{\infty} d y 12 \kappa f_{1}(y) \partial_{y} f_{2}(y)=-\frac{3}{16} \kappa^{2}[48 \mathcal{C}-\pi(\pi+12 \log 2)], \\
& \tau_{30}=-\int_{1}^{\infty} d y 72 \kappa^{2}\left[\frac{1}{y^{3}} b_{6}(y)\right]=\frac{9}{2} \kappa^{2}(\log 2)^{2}, \\
& \tau_{31}=-\int_{1}^{\infty} d y \frac{432 \kappa^{3}}{y^{3}}\left[y \partial_{y} b_{6}(y)+2 b_{6}(y)\right]=-\frac{9}{2} \kappa^{3}\left(\pi^{2}-24 \log ^{2} 2\right), \\
& \tau_{32}=\int_{1}^{\infty} d y 12 \kappa\left[\frac{1}{2 y^{2}} \partial_{y} b_{1}(y)-f_{1}(y) \partial_{y} f_{1}(y)\right]=-\frac{1}{64} \kappa[48 \mathcal{C}+\pi(\pi-24 \log 2)], \\
& \tau_{33}=-\int_{1}^{\infty} d y 12 \kappa f_{2}(y) \partial_{y} f_{2}(y)=54 \kappa^{3}(\log 2)^{2}, \\
& \tau_{34}=\int_{1}^{\infty} d y 432 \kappa^{3} \partial_{y} b_{5}(y)=9 \kappa^{3}\left[\pi^{2}-6 \log 2(\log 2-2)\right], \\
& \tau_{35}=\int_{1}^{\infty} d y 72 \kappa^{2}\left[\frac{1}{y^{3}} b_{6}(y)\right]=-\frac{9}{2} \kappa^{2}(\log 2)^{2}, \\
& \tau_{36}=\int_{1}^{\infty} d y 72 \kappa^{2}\left[\frac{1}{2 y^{2}} \partial_{y} b_{6}(y)\right]=\frac{3}{8} \kappa^{2}\left[\pi^{2}-12(\log 2)^{2}\right], \\
& \tau_{37}=-\int_{1}^{\infty} d y \frac{216 \kappa^{3}}{y^{3}}\left[y \partial_{y} b_{5}(y)+4 b_{5}(y)\right]=\frac{9}{2} \kappa^{3}\left[72-\pi^{2}+30(\log 2-2) \log 2\right],
\end{aligned}
$$




$$
\begin{aligned}
& \tau_{38}=-\int_{1}^{\infty} d y 432 \kappa^{3}\left[\frac{1}{y^{3}} b_{7}(y)-\frac{1}{y^{3}} b_{5}(y)\right]=\kappa^{3}\left(324-135 \log ^{2} 2+162\right), \\
& \tau_{39}=\int_{1}^{\infty} d y \frac{216 \kappa^{3}}{y^{2}}\left[\partial_{y} b_{5}(y)-\partial_{y} b_{7}(y)\right]=-\frac{27}{4} \kappa^{3}\left[24-\pi^{2}-16 \log 2(\log 2-2)\right], \\
& \tau_{40}=-\int_{1}^{\infty} d y 432 \kappa^{3}\left[\partial_{y} b_{5}(y)-\partial_{y} b_{7}(y)\right]=-\frac{27}{2} \kappa^{3}\left[\pi^{2}-4 \log 2(\log 2-4)\right]
\end{aligned}
$$

where the Catalan's constant $\mathcal{C} \approx 0.915966$.

\section{Acknowledgments}

We would like to thank Umut Gürsoy, Dmitri Kharzeev, Nathan Kleeorin, Shu Lin, Igor Rogachevskii, Andrey Sadofyev, Ho-Ung Yee for useful discussions. YB would like to thank the hospitality of the Department of Physics at Ben-Gurion University of the Negev where this work was initialised and finalised. YB was supported by the Fundamental Research Funds for the Central Universities under grant No.122050205032 and the Natural Science Foundation of China (NSFC) under the grant No.11705037. TD and ML were supported by the Israeli Science Foundation (ISF) grant \#1635/16 and the BSF grants \#2012124 and \#2014707.

Open Access. This article is distributed under the terms of the Creative Commons Attribution License (CC-BY 4.0), which permits any use, distribution and reproduction in any medium, provided the original author(s) and source are credited.

\section{References}

[1] L. Landau and E. Lifshitz, Fluid Mechanics, Volume 6, Elsevier Science, (2013).

[2] D. Forster, Hydrodynamic fluctuations, broken symmetry, and correlation functions, CRC Press, (1975).

[3] M. Lublinsky and E. Shuryak, How much entropy is produced in strongly coupled quark-gluon Plasma (sQGP) by dissipative effects?, Phys. Rev. C 76 (2007) 021901 [arXiv:0704.1647] [INSPIRE].

[4] M. Lublinsky and E. Shuryak, Improved Hydrodynamics from the AdS/CFT, Phys. Rev. D 80 (2009) 065026 [arXiv: 0905.4069] [INSPIRE].

[5] Y. Bu and M. Lublinsky, All order linearized hydrodynamics from fluid-gravity correspondence, Phys. Rev. D 90 (2014) 086003 [arXiv: 1406.7222] [INSPIRE].

[6] Y. Bu and M. Lublinsky, Linearized fluid/gravity correspondence: from shear viscosity to all order hydrodynamics, JHEP 11 (2014) 064 [arXiv:1409.3095] [INSPIRE].

[7] Y. Bu and M. Lublinsky, Linearly resummed hydrodynamics in a weakly curved spacetime, JHEP 04 (2015) 136 [arXiv: 1502.08044] [InSPIRE].

[8] Y. Bu, M. Lublinsky and A. Sharon, Hydrodynamics dual to Einstein-Gauss-Bonnet gravity: all-order gradient resummation, JHEP 06 (2015) 162 [arXiv:1504.01370] [INSPIRE].

[9] I. Müller, Zum paradoxon der wärmeleitungstheorie, Z. Phys. 198 (1967) 329. 
[10] W. Israel, Nonstationary irreversible thermodynamics: A causal relativistic theory, Annals Phys. 100 (1976) 310 [INSPIRE].

[11] W. Israel and J. Stewart, Thermodynamics of nonstationary and transient effects in a relativistic gas, Phys. Lett. A 58 (1976) 213.

[12] W. Israel and J.M. Stewart, Transient relativistic thermodynamics and kinetic theory, Annals Phys. 118 (1979) 341 [INSPIRE].

[13] M.P. Heller, R.A. Janik and P. Witaszczyk, Hydrodynamic Gradient Expansion in Gauge Theory Plasmas, Phys. Rev. Lett. 110 (2013) 211602 [arXiv:1302.0697] [INSPIRE].

[14] M.P. Heller and M. Spaliński, Hydrodynamics Beyond the Gradient Expansion: Resurgence and Resummation, Phys. Rev. Lett. 115 (2015) 072501 [arXiv:1503.07514] [INSPIRE].

[15] S. Grozdanov and N. Kaplis, Constructing higher-order hydrodynamics: The third order, Phys. Rev. D 93 (2016) 066012 [arXiv:1507.02461] [InSPIRE].

[16] P. Romatschke, Do nuclear collisions create a locally equilibrated quark-gluon plasma?, Eur. Phys. J. C 77 (2017) 21 [arXiv:1609.02820] [InSPIRE].

[17] P. Romatschke, Relativistic Fluid Dynamics Far From Local Equilibrium, Phys. Rev. Lett. 120 (2018) 012301 [arXiv: 1704.08699] [INSPIRE].

[18] M. Spaliński, On the hydrodynamic attractor of Yang-Mills plasma, Phys. Lett. B 776 (2018) 468 [arXiv:1708.01921] [INSPIRE].

[19] P. Romatschke, Relativistic Hydrodynamic Attractors with Broken Symmetries: Non-Conformal and Non-Homogeneous, JHEP 12 (2017) 079 [arXiv:1710.03234] [INSPIRE].

[20] W. Florkowski, M.P. Heller and M. Spaliński, New theories of relativistic hydrodynamics in the LHC era, Rept. Prog. Phys. 81 (2018) 046001 [arXiv:1707.02282] [INSPIRE].

[21] G.S. Denicol and J. Noronha, Hydrodynamic attractor and the fate of perturbative expansions in Gubser flow, arXiv:1804.04771 [INSPIRE].

[22] A. Behtash, C.N. Cruz-Camacho and M. Martinez, Far-from-equilibrium attractors and nonlinear dynamical systems approach to the Gubser flow, Phys. Rev. D 97 (2018) 044041 [arXiv: 1711.01745] [INSPIRE].

[23] A. Behtash, S. Kamata, M. Martinez and C.N. Cruz-Camacho, Non-perturbative rheological behavior of a far-from-equilibrium expanding plasma, arXiv:1805.07881 [INSPIRE].

[24] Y. Bu, M. Lublinsky and A. Sharon, Anomalous transport from holography: Part I, JHEP 11 (2016) 093 [arXiv: 1608.08595] [INSPIRE].

[25] Y. Bu, M. Lublinsky and A. Sharon, Anomalous transport from holography: Part II, Eur. Phys. J. C 77 (2017) 194 [arXiv:1609.09054] [INSPIRE].

[26] V.A. Kuzmin, V.A. Rubakov and M.E. Shaposhnikov, On the Anomalous Electroweak Baryon Number Nonconservation in the Early Universe, Phys. Lett. 155B (1985) 36 [INSPIRE].

[27] A. Vilenkin and D.A. Leahy, Parity non-conservation and the origin of cosmic magnetic fields, Astrophys. J. 254 (1982) 77 [INSPIRE].

[28] V.A. Rubakov and M.E. Shaposhnikov, Electroweak baryon number nonconservation in the early universe and in high-energy collisions, Usp. Fiz. Nauk 166 (1996) 493 [hep-ph/9603208] [INSPIRE]. 
[29] D. Grasso and H.R. Rubinstein, Magnetic fields in the early universe, Phys. Rept. 348 (2001) 163 [astro-ph/0009061] [INSPIRE].

[30] M. Giovannini, The magnetized universe, Int. J. Mod. Phys. D 13 (2004) 391 [astro-ph/0312614] [INSPIRE].

[31] D.E. Kharzeev, Topology, magnetic field and strongly interacting matter, Ann. Rev. Nucl. Part. Sci. 65 (2015) 193 [arXiv:1501.01336] [INSPIRE].

[32] D.E. Kharzeev, The Chiral Magnetic Effect and Anomaly-Induced Transport, Prog. Part. Nucl. Phys. 75 (2014) 133 [arXiv:1312.3348] [InSPIRE].

[33] X.-G. Huang, Electromagnetic fields and anomalous transports in heavy-ion collisions - A pedagogical review, Rept. Prog. Phys. 79 (2016) 076302 [arXiv:1509.04073] [INSPIRE].

[34] ALICE collaboration, Charge-dependent flow and the search for the chiral magnetic wave in Pb-Pb collisions at $\sqrt{s_{\mathrm{NN}}}=2.76$ TeV, Phys. Rev. C 93 (2016) 044903 [arXiv:1512.05739] [INSPIRE].

[35] CMS collaboration, Observation of charge-dependent azimuthal correlations in $p-P b$ collisions and its implication for the search for the chiral magnetic effect, Phys. Rev. Lett. 118 (2017) 122301 [arXiv: 1610.00263] [INSPIRE].

[36] CMS collaboration, Constraints on the chiral magnetic effect using charge-dependent azimuthal correlations in $\mathrm{pPb}$ and $\mathrm{PbPb}$ collisions at the CERN Large Hadron Collider, Phys. Rev. C 97 (2018) 044912 [arXiv:1708.01602] [INSPIRE].

[37] CMS collaboration, Challenges to the chiral magnetic wave using charge-dependent azimuthal anisotropies in $\mathrm{pPb}$ and $\mathrm{PbPb}$ collisions at $\sqrt{s_{\mathrm{NN}}}=5.02 \mathrm{TeV}$, arXiv:1708.08901 [INSPIRE].

[38] V. Koch et al., Status of the chiral magnetic effect and collisions of isobars, Chin. Phys. C 41 (2017) 072001 [arXiv: 1608.00982] [INSPIRE].

[39] Z.K. Liu et al., Discovery of a Three-Dimensional Topological Dirac Semimetal, $\mathrm{Na}_{3} \mathrm{Bi}$, Science 343 (2015) 864.

[40] B.Q. Lv et al., Experimental discovery of Weyl semimetal TaAs, Phys. Rev. X 5 (2015) 031013 [arXiv: 1502.04684] [INSPIRE].

[41] S.Y. Xu et al., Discovery of a Weyl Fermion semimetal and topological Fermi arcs, Science 349 (2015) 613 [inSPIRE].

[42] O. Vafek and A. Vishwanath, Dirac Fermions in Solids: From High-Tc cuprates and Graphene to Topological Insulators and Weyl Semimetals, Ann. Rev. Condensed Matter Phys. 5 (2014) 83 [arXiv: 1306.2272] [INSPIRE].

[43] Q. Li et al., Observation of the chiral magnetic effect in ZrTe, Nature Phys. 12 (2016) 550 [arXiv:1412.6543] [INSPIRE].

[44] X. Huang et al., Observation of the Chiral-Anomaly-Induced Negative Magnetoresistance in $3 D$ Weyl Semimetal TaAs, Phys. Rev. X 5 (2015) 031023 [arXiv:1503.01304] [InSPIRE].

[45] H. Li et al., Negative Magnetoresistance in Dirac Semimetal $\mathrm{Cd}_{3} A s_{2}$, Nat. Commun. 7 (2016) 10301 [arXiv: 1507.06470].

[46] K. Landsteiner, E. Megias and F. Pena-Benitez, Anomalous Transport from Kubo Formulae, Lect. Notes Phys. 871 (2013) 433 [arXiv:1207.5808]. 
[47] K. Landsteiner, Y. Liu and Y.-W. Sun, Negative magnetoresistivity in chiral fluids and holography, JHEP 03 (2015) 127 [arXiv:1410.6399] [INSPIRE].

[48] A. Jimenez-Alba, K. Landsteiner, Y. Liu and Y.-W. Sun, Anomalous magnetoconductivity and relaxation times in holography, JHEP 07 (2015) 117 [arXiv: 1504.06566] [INSPIRE].

[49] K. Landsteiner and Y. Liu, The holographic Weyl semi-metal, Phys. Lett. B 753 (2016) 453 [arXiv: 1505.04772] [INSPIRE].

[50] A. Vilenkin, Equilibrium parity violating current in a magnetic field, Phys. Rev. D 22 (1980) 3080 [INSPIRE].

[51] K. Fukushima, D.E. Kharzeev and H.J. Warringa, The Chiral Magnetic Effect, Phys. Rev. D 78 (2008) 074033 [arXiv:0808.3382] [InSPIRE].

[52] D.E. Kharzeev and H.J. Warringa, Chiral Magnetic conductivity, Phys. Rev. D 80 (2009) 034028 [arXiv: 0907.5007] [INSPIRE].

[53] K. Fukushima, D.E. Kharzeev and H.J. Warringa, Real-time dynamics of the Chiral Magnetic Effect, Phys. Rev. Lett. 104 (2010) 212001 [arXiv:1002.2495] [INSPIRE].

[54] D. Hou, H. Liu and H.-c. Ren, Some Field Theoretic Issues Regarding the Chiral Magnetic Effect, JHEP 05 (2011) 046 [arXiv: 1103.2035] [INSPIRE].

[55] D. Satow and H.-U. Yee, Chiral Magnetic Effect at Weak Coupling with Relaxation Dynamics, Phys. Rev. D 90 (2014) 014027 [arXiv: 1406.1150] [InSPIRE].

[56] H.-U. Yee, Chiral Magnetic and Vortical Effects in Higher Dimensions at Weak Coupling, Phys. Rev. D 90 (2014) 065021 [arXiv: 1406.3584] [INSPIRE].

[57] A. Jimenez-Alba and H.-U. Yee, Second order transport coefficient from the chiral anomaly at weak coupling: Diagrammatic resummation, Phys. Rev. D 92 (2015) 014023 [arXiv: 1504.05866] [INSPIRE].

[58] G.M. Newman, Anomalous hydrodynamics, JHEP 01 (2006) 158 [hep-ph/0511236] [INSPIRE].

[59] H.-U. Yee, Holographic Chiral Magnetic Conductivity, JHEP 11 (2009) 085 [arXiv: 0908.4189] [INSPIRE].

[60] A. Rebhan, A. Schmitt and S.A. Stricker, Anomalies and the chiral magnetic effect in the Sakai-Sugimoto model, JHEP 01 (2010) 026 [arXiv:0909.4782] [INSPIRE].

[61] Y. Matsuo, S.-J. Sin, S. Takeuchi and T. Tsukioka, Magnetic conductivity and Chern-Simons Term in Holographic Hydrodynamics of Charged AdS Black Hole, JHEP 04 (2010) 071 [arXiv: 0910.3722] [INSPIRE].

[62] A. Gorsky, P.N. Kopnin and A.V. Zayakin, On the Chiral Magnetic Effect in Soft-Wall AdS/QCD, Phys. Rev. D 83 (2011) 014023 [arXiv:1003.2293] [InSPIRE].

[63] V.A. Rubakov, On chiral magnetic effect and holography, arXiv:1005.1888 [INSPIRE].

[64] A. Gynther, K. Landsteiner, F. Pena-Benitez and A. Rebhan, Holographic Anomalous Conductivities and the Chiral Magnetic Effect, JHEP 02 (2011) 110 [arXiv:1005.2587] [INSPIRE].

[65] I. Amado, K. Landsteiner and F. Pena-Benitez, Anomalous transport coefficients from Kubo formulas in Holography, JHEP 05 (2011) 081 [arXiv: 1102.4577] [InSPIRE].

[66] T. Kalaydzhyan and I. Kirsch, Fluid/gravity model for the chiral magnetic effect, Phys. Rev. Lett. 106 (2011) 211601 [arXiv:1102.4334] [INSPIRE]. 
[67] C. Hoyos, T. Nishioka and A. O'Bannon, A Chiral Magnetic Effect from AdS/CFT with Flavor, JHEP 10 (2011) 084 [arXiv: 1106.4030] [INSPIRE].

[68] Y.-P. Hu, P. Sun and J.-H. Zhang, Hydrodynamics with conserved current via AdS/CFT correspondence in the Maxwell-Gauss-Bonnet gravity, Phys. Rev. D 83 (2011) 126003 [arXiv:1103.3773] [INSPIRE].

[69] Y.-P. Hu and C. Park, Chern-Simons effect on the dual hydrodynamics in the Maxwell-Gauss-Bonnet gravity, Phys. Lett. B 714 (2012) 324 [arXiv:1112.4227] [INSPIRE].

[70] X. Bai, Y.-P. Hu, B.-H. Lee and Y.-L. Zhang, Holographic Charged Fluid with Anomalous Current at Finite Cutoff Surface in Einstein-Maxwell Gravity, JHEP 11 (2012) 054 [arXiv:1207.5309] [INSPIRE].

[71] S. Lin and H.-U. Yee, Out-of-Equilibrium Chiral Magnetic Effect at Strong Coupling, Phys. Rev. D 88 (2013) 025030 [arXiv: 1305.3949] [INSPIRE].

[72] I. Iatrakis, S. Lin and Y. Yin, The anomalous transport of axial charge: topological vs non-topological fluctuations, JHEP 09 (2015) 030 [arXiv:1506.01384] [INSPIRE].

[73] S. Grozdanov and N. Poovuttikul, Universality of anomalous conductivities in theories with higher-derivative holographic duals, JHEP 09 (2016) 046 [arXiv: 1603.08770] [INSPIRE].

[74] M. Ammon, M. Kaminski, R. Koirala, J. Leiber and J. Wu, Quasinormal modes of charged magnetic black branes 85 chiral magnetic transport, JHEP 04 (2017) 067 [arXiv: 1701.05565] [INSPIRE].

[75] J.M. Maldacena, The large $N$ limit of superconformal field theories and supergravity, Int. $J$. Theor. Phys. 38 (1999) 1113 [hep-th/9711200] [INSPIRE].

[76] S.S. Gubser, I.R. Klebanov and A.M. Polyakov, Gauge theory correlators from noncritical string theory, Phys. Lett. B 428 (1998) 105 [hep-th/9802109] [INSPIRE].

[77] E. Witten, Anti-de Sitter space and holography, Adv. Theor. Math. Phys. 2 (1998) 253 [hep-th/9802150] [INSPIRE].

[78] D.T. Son and P. Surowka, Hydrodynamics with Triangle Anomalies, Phys. Rev. Lett. 103 (2009) 191601 [arXiv:0906.5044] [INSPIRE].

[79] A.V. Sadofyev and M.V. Isachenkov, The chiral magnetic effect in hydrodynamical approach, Phys. Lett. B 697 (2011) 404 [arXiv: 1010.1550] [INSPIRE].

[80] D.T. Son and N. Yamamoto, Berry Curvature, Triangle Anomalies and the Chiral Magnetic Effect in Fermi Liquids, Phys. Rev. Lett. 109 (2012) 181602 [arXiv:1203.2697] [INSPIRE].

[81] M.A. Stephanov and Y. Yin, Chiral Kinetic Theory, Phys. Rev. Lett. 109 (2012) 162001 [arXiv:1207.0747] [INSPIRE].

[82] D.T. Son and N. Yamamoto, Kinetic theory with Berry curvature from quantum field theories, Phys. Rev. D 87 (2013) 085016 [arXiv:1210.8158] [InSPIRE].

[83] J.-H. Gao, Z.-T. Liang, S. Pu, Q. Wang and X.-N. Wang, Chiral Anomaly and Local Polarization Effect from Quantum Kinetic Approach, Phys. Rev. Lett. 109 (2012) 232301 [arXiv: 1203.0725] [INSPIRE].

[84] J.-W. Chen, S. Pu, Q. Wang and X.-N. Wang, Berry Curvature and Four-Dimensional Monopoles in the Relativistic Chiral Kinetic Equation, Phys. Rev. Lett. 110 (2013) 262301 [arXiv:1210.8312] [INSPIRE]. 
[85] P.V. Buividovich, M.N. Chernodub, E.V. Luschevskaya and M.I. Polikarpov, Numerical evidence of chiral magnetic effect in lattice gauge theory, Phys. Rev. D 80 (2009) 054503 [arXiv: 0907.0494] [INSPIRE].

[86] M. Abramczyk, T. Blum, G. Petropoulos and R. Zhou, Chiral magnetic effect in 2+1 flavor $Q C D+Q E D$, PoS (LAT2009) 181 (2009) [arXiv:0911.1348] [INSPIRE].

[87] K. Fukushima, D.E. Kharzeev and H.J. Warringa, Electric-current Susceptibility and the Chiral Magnetic Effect, Nucl. Phys. A 836 (2010) 311 [arXiv:0912.2961] [InSPIRE].

[88] V.V. Braguta, P.V. Buividovich, T. Kalaydzhyan, S.V. Kuznetsov and M.I. Polikarpov, The Chiral Magnetic Effect and chiral symmetry breaking in $\mathrm{SU}(3)$ quenched lattice gauge theory, Phys. Atom. Nucl. 75 (2012) 488 [arXiv:1011.3795] [INSPIRE].

[89] A. Yamamoto, Chiral magnetic effect in lattice QCD with a chiral chemical potential, Phys. Rev. Lett. 107 (2011) 031601 [arXiv: 1105.0385] [INSPIRE].

[90] V. Braguta, M.N. Chernodub, K. Landsteiner, M.I. Polikarpov and M.V. Ulybyshev, Numerical evidence of the axial magnetic effect, Phys. Rev. D 88 (2013) 071501 [arXiv: 1303.6266] [INSPIRE].

[91] N. Yamamoto, Generalized Bloch theorem and chiral transport phenomena, Phys. Rev. D 92 (2015) 085011 [arXiv: 1502.01547] [INSPIRE].

[92] M.A. Zubkov, Absence of equilibrium chiral magnetic effect, Phys. Rev. D 93 (2016) 105036 [arXiv: 1605. 08724] [INSPIRE].

[93] D.T. Son and A.R. Zhitnitsky, Quantum anomalies in dense matter, Phys. Rev. D 70 (2004) 074018 [hep-ph/0405216] [INSPIRE].

[94] M.A. Metlitski and A.R. Zhitnitsky, Anomalous axion interactions and topological currents in dense matter, Phys. Rev. D 72 (2005) 045011 [hep-ph/0505072] [INSPIRE].

[95] D.E. Kharzeev and H.-U. Yee, Chiral Magnetic Wave, Phys. Rev. D 83 (2011) 085007 [arXiv: 1012.6026] [INSPIRE].

[96] Y. Bu, T. Demircik and M. Lublinsky, Gradient resummation for nonlinear chiral transport: an insight from holography, arXiv:1807.11908 [INSPIRE].

[97] M. Joyce and M.E. Shaposhnikov, Primordial magnetic fields, right-handed electrons and the Abelian anomaly, Phys. Rev. Lett. 79 (1997) 1193 [astro-ph/9703005] [INSPIRE].

[98] A. Boyarsky, J. Fröhlich and O. Ruchayskiy, Self-consistent evolution of magnetic fields and chiral asymmetry in the early Universe, Phys. Rev. Lett. 108 (2012) 031301 [arXiv: 1109.3350] [INSPIRE].

[99] C. Manuel and J.M. Torres-Rincon, Dynamical evolution of the chiral magnetic effect: Applications to the quark-gluon plasma, Phys. Rev. D 92 (2015) 074018 [arXiv: 1501.07608] [INSPIRE].

[100] A. Boyarsky, J. Fröhlich and O. Ruchayskiy, Magnetohydrodynamics of Chiral Relativistic Fluids, Phys. Rev. D 92 (2015) 043004 [arXiv: 1504.04854] [INSPIRE].

[101] Y. Hirono, D. Kharzeev and Y. Yin, Self-similar inverse cascade of magnetic helicity driven by the chiral anomaly, Phys. Rev. D 92 (2015) 125031 [arXiv: 1509.07790] [InSPIRE].

[102] A. Avdoshkin, V.P. Kirilin, A.V. Sadofyev and V.I. Zakharov, On consistency of hydrodynamic approximation for chiral media, Phys. Lett. B 755 (2016) 1 [arXiv: 1402 .3587] [INSPIRE]. 
[103] J.-W. Chen, T. Ishii, S. Pu and N. Yamamoto, Nonlinear Chiral Transport Phenomena, Phys. Rev. D 93 (2016) 125023 [arXiv:1603.03620] [InSPIRE].

[104] E.V. Gorbar, I.A. Shovkovy, S. Vilchinskii, I. Rudenok, A. Boyarsky and O. Ruchayskiy, Anomalous Maxwell equations for inhomogeneous chiral plasma, Phys. Rev. D 93 (2016) 105028 [arXiv: 1603.03442] [INSPIRE].

[105] O.F. Dayi and E. Kilinçarslan, Nonlinear Chiral Plasma Transport in Rotating Coordinates, Phys. Rev. D 96 (2017) 043514 [arXiv: 1705.01267] [INSPIRE].

[106] Y. Hidaka, S. Pu and D.-L. Yang, Nonlinear Responses of Chiral Fluids from Kinetic Theory, Phys. Rev. D 97 (2018) 016004 [arXiv: 1710.00278] [InSPIRE].

[107] D.E. Kharzeev and H.-U. Yee, Anomalies and time reversal invariance in relativistic hydrodynamics: the second order and higher dimensional formulations, Phys. Rev. D 84 (2011) 045025 [arXiv:1105.6360] [INSPIRE].

[108] E. Megias and F. Pena-Benitez, Holographic Gravitational Anomaly in First and Second Order Hydrodynamics, JHEP 05 (2013) 115 [arXiv:1304.5529] [INSPIRE].

[109] U. Gürsoy and J. Tarrio, Horizon universality and anomalous conductivities, JHEP 10 (2015) 058 [arXiv: 1410.1306] [INSPIRE].

[110] U. Gürsoy and A. Jansen, (Non)renormalization of Anomalous Conductivities and Holography, JHEP 10 (2014) 092 [arXiv: 1407.3282] [INSPIRE].

[111] Y. Bu, M. Lublinsky and A. Sharon, U(1) current from the AdS/CFT: diffusion, conductivity and causality, JHEP 04 (2016) 136 [arXiv:1511.08789] [INSPIRE].

[112] G. Basar and G.V. Dunne, Hydrodynamics, resurgence and transasymptotics, Phys. Rev. D 92 (2015) 125011 [arXiv: 1509.05046] [INSPIRE].

[113] Y. Bu, R.-G. Cai, Q. Yang and Y.-L. Zhang, Holographic Charged Fluid with Chiral Electric Separation Effect, JHEP 09 (2018) 083 [arXiv: 1803.08389] [INSPIRE].

[114] S. Li and H.-U. Yee, Relaxation times for chiral transport phenomena and spin polarization in a strongly coupled plasma, Phys. Rev. D 98 (2018) 056018 [arXiv:1805.04057] [INSPIRE].

[115] R.C. Myers, A.O. Starinets and R.M. Thomson, Holographic spectral functions and diffusion constants for fundamental matter, JHEP 11 (2007) 091 [arXiv:0706.0162] [INSPIRE].

[116] S. Pu, S.-Y. Wu and D.-L. Yang, Chiral Hall Effect and Chiral Electric Waves, Phys. Rev. D 91 (2015) 025011 [arXiv: 1407.3168] [InSPIRE].

[117] Y. Bu, T. Demircik and M. Lublinsky, Nonlinear chiral transport from holography: strong field limit, in preparation.

[118] S. Bhattacharyya, V.E. Hubeny, S. Minwalla and M. Rangamani, Nonlinear Fluid Dynamics from Gravity, JHEP 02 (2008) 045 [arXiv:0712.2456] [INSPIRE].

[119] J. Erdmenger, M. Haack, M. Kaminski and A. Yarom, Fluid dynamics of R-charged black holes, JHEP 01 (2009) 055 [arXiv:0809.2488] [INSPIRE].

[120] N. Banerjee, J. Bhattacharya, S. Bhattacharyya, S. Dutta, R. Loganayagam and P. Surowka, Hydrodynamics from charged black branes, JHEP 01 (2011) 094 [arXiv:0809.2596] [INSPIRE]. 